\title{
Serine-ubiquitination regulates Golgi morphology and the secretory pathway upon Legionella infection
}

\author{
Yaobin Liu ${ }^{1,2}$, Rukmini Mukherjee (iD ${ }^{1,2}$, Florian Bonn ${ }^{1}$, Thomas Colby ${ }^{3}$, Ivan Matic $\mathbb{D i D}^{3,4}$, Marius Glogger $^{5}$, Mike Heilemann (D) $^{5}$ and \\ Ivan Dikic (iD) ${ }^{1,2}$ 凶
}

(c) The Author(s) 2021

SidE family of Legionella effectors catalyze non-canonical phosphoribosyl-linked ubiquitination (PR-ubiquitination) of host proteins during bacterial infection. SdeA localizes predominantly to ER and partially to the Golgi apparatus, and mediates serine ubiquitination of multiple ER and Golgi proteins. Here we show that SdeA causes disruption of Golgi integrity due to its ubiquitin ligase activity. The Golgi linking proteins GRASP55 and GRASP65 are PR-ubiquitinated on multiple serine residues, thus preventing their ability to cluster and form oligomeric structures. In addition, we found that the functional consequence of Golgi disruption is not linked to the recruitment of Golgi membranes to the growing Legionella-containing vacuoles. Instead, it affects the host secretory pathway. Taken together, our study sheds light on the Golgi manipulation strategy by which Legionella hijacks the secretory pathway and promotes bacterial infection.

Cell Death \& Differentiation (2021) 28:2957-2969; https://doi.org/10.1038/s41418-021-00830-y

\section{INTRODUCTION}

Ubiquitination is a post-translational modification that is conserved from yeast to mammals. The catalysis of canonical ubiquitination is regulated via a well-known E1-E2-E3 threeenzyme cascade in an ATP dependent manner [1]. Protein ubiquitination virtually regulates every cellular processes, including protein stability, protein trafficking, immunity, and DNA repair [2-5].

Consistent with the critical roles of ubiquitination in cellular processes, emerging evidence indicates that pathogens hijack the ubiquitination machinery for efficient invasion [6-8]. Various studies have revealed that effectors of the SidE family (SdeA, SdeB, SdeC and SidE) catalyze an NAD ${ }^{+}$-dependent, ATPindependent type of ubiquitination without the need of E2 and E3 enzymes [9, 10]. Moreover, unlike the conventional ubiquitination that occurs on lysine residues of substrate proteins, SidE family effectors catalyze the conjugation of Ub via a phosphoribosyl moiety to serine residues of host substrate proteins by a two-domain catalytic relay: a mono ADP-ribosyl transferase (mART) domain and a phosphodiesterase (PDE) domain [11-14].

Phosphoribosyl (PR)-linked ubiquitination can be reversed by DupA, a deubiquitinase with specific affinity for PR-ubiquitinated substrates [15]. In our previous study, we used the catalytically mutant DupA as a bait to identify targets of SdeA. Besides ERrelated substrates, we also identified proteins related to other cellular pathways, including Golgi proteins, mitochondrial proteins and components of the autophagy machinery [15]. However, the biological functions of PR-ubiquitination of these proteins remained unclear. In this study, we made use of biochemical and microbiological approaches to characterize the PRubiquitination of Golgi tethering proteins GRASP55 and GRASP65 by SdeA. We also provide explanations for the Golgi morphological regulation by the PR-ubiquitination of these proteins. Moreover, we demonstrate that PR-ubiquitination regulates the host cellular secretory pathway during bacterial infection.

\section{RESULTS}

SdeA is targeted to the ER and Golgi via its carboxyl terminus Previous structural and biochemical studies have revealed the structure of SdeA catalytic core, and the mechanism by which SdeA ubiquitinates substrates is well established [11-13]. However, the function of the carboxyl terminal (CT) coiled-coil region, remained unknown (Fig. 1A). Previous reports suggested that coiled-coil domains are required for membrane localization of many Salmonella type III effectors [16]. In view of that SdeA colocalizes with ER protein calnexin and ubiquitinates many ER proteins, such as RTN4 and FAM134B [15, 17], we hypothesized that CT domain of SdeA is responsible for its membrane association. To test this, we first investigated the ER localization of wild-type SdeA and truncated Sde $A^{1-972}$ mutant lacking the last part of the $\mathrm{C}$-terminal region (Fig. $1 \mathrm{~A}$ ). We observed that ectopically expressed SdeA colocalized with ER protein Calnexin in COS7 cells, consistently with a previous study [18]. However the truncated SdeA did not co-localize with Calnexin but showed a rather cytosolic distribution (Fig. 1B). In addition, we observed that part of SdeA was densely localized close to the nucleus in cells (Fig. 1B). Staining with the Golgi marker GM130 revealed that this

\footnotetext{
${ }^{1}$ Institute of Biochemistry II, School of Medicine, Goethe University Frankfurt, Frankfurt am Main, Germany. ${ }^{2}$ Buchmann Institute for Molecular Life Sciences, Goethe University

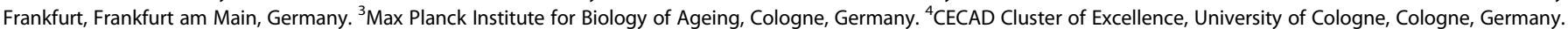
${ }^{5}$ Institute for Physical and Theoretical Chemistry, Goethe-University Frankfurt, Frankfurt am Main, Germany. ${ }^{凶}$ email: dikic@biochem2.uni-frankfurt.de Edited by G. Melino
} 
A

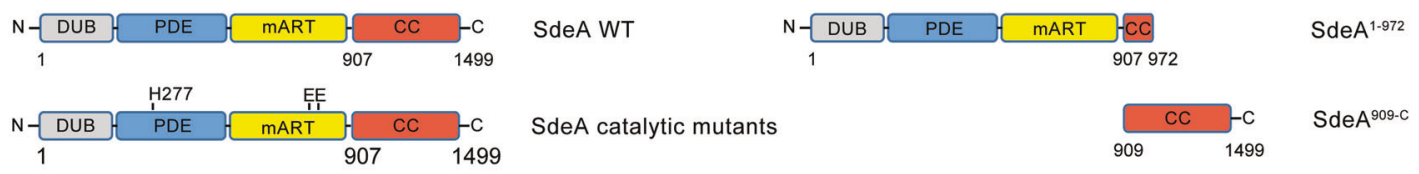

B

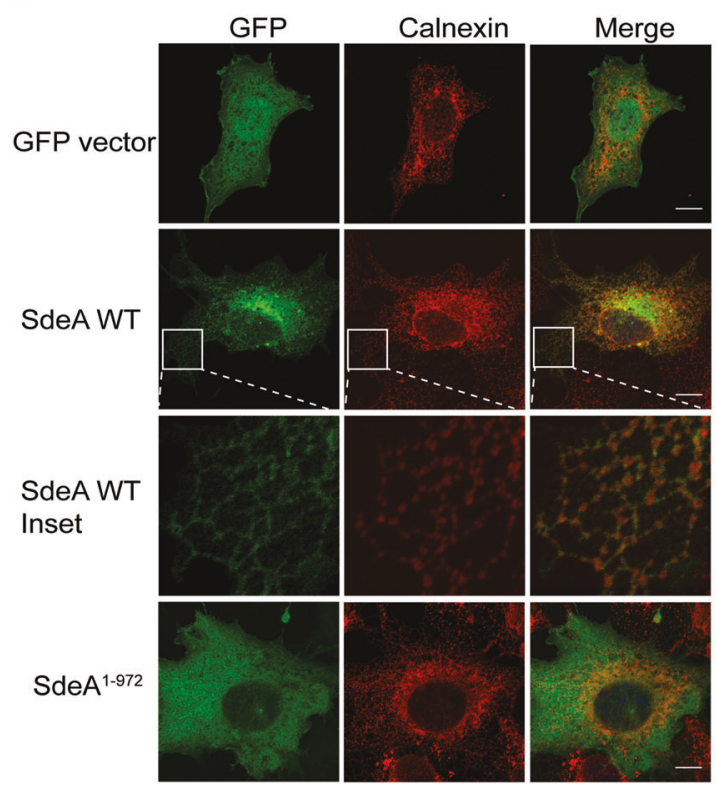

C

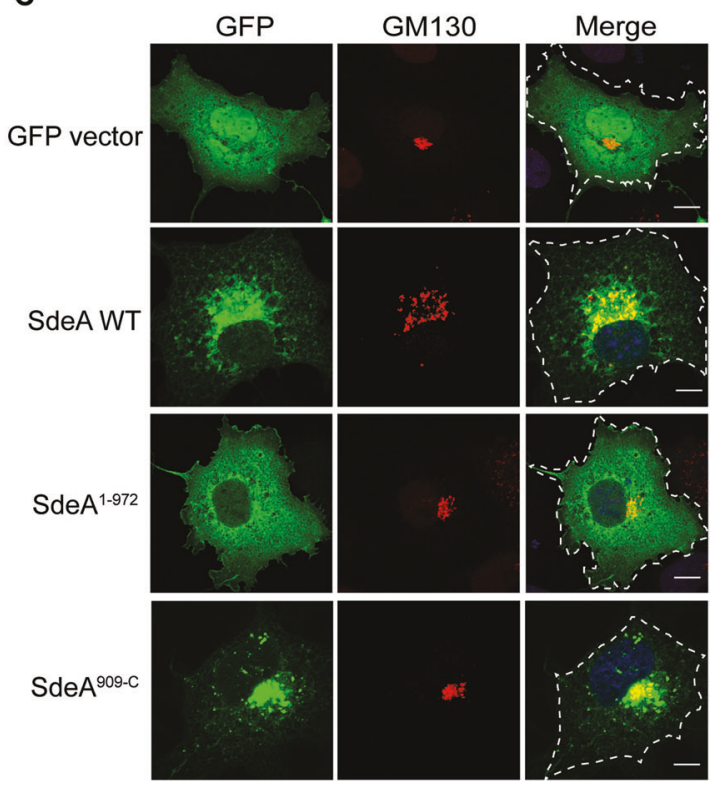

Fig. 1 SdeA partially localizes to the Golgi. A Schematic diagrams of full-length wild-type SdeA, SdeA catalytic mutant SdeA H277A or SdeA EE/AA, truncated SdeA ${ }^{1-972}$ and SdeA ${ }^{909-C}$. B Confocal images showing the colocalization of SdeA (green) with ER protein Calnexin (red). COS7 cells were transfected with plasmids encoding GFP-tagged wild type SdeA or truncated mutant. Cells were cultured for $24 \mathrm{~h}$ after transfection, then fixed, permeabilized, and stained with Calnexin antibody and visualized using confocal microscope. Scale bars, $10 \mu \mathrm{m}$. C Confocal images showing the colocalization of SdeA (green) with Golgi protein GM130 (red). Cells were cultured for $24 \mathrm{~h}$ after transfection, then fixed, permeabilized, and ultimately stained with GM130 antibody and visualized using confocal microscope. Scale bars, $10 \mu \mathrm{m}$.

part of SdeA colocalized with the Golgi apparatus, while the truncated mutant SdeA ${ }^{1-972}$ did not (Fig. 1C). We confirmed that the C-terminus region of SdeA is necessary to its Golgi localization by expressing the N-terminal-truncated Sde $A^{909-C}$ in cells (Fig. 1C). This data suggests that C-terminal part of SdeA is critical for its ER as well as Golgi membrane localization.

\section{SdeA induces disruption of Golgi structure}

To investigate whether Golgi localization of SdeA is critical for its ligase function, we co-expressed wild-type SdeA or the truncated mutant SdeA $\mathrm{A}^{1-972}$ with its known Golgi associated substrate Rab33b [9]. Western blot analysis showed that the truncated form of SdeA could not ubiquitinate Rab33b even though it was able to ADP-ribosylate ubiquitin (Fig.S1A). This data suggests that the C-terminus region of SdeA is critical not only for its localization but also for its ability to ubiquitinate Golgi proteins. During our localization studies, we observed that expression of wild-type SdeA, but not the CT-truncated mutants, results in dispersed GM130 staining (Fig. 1C). This implicates an effect of SdeA activity on the structural organization of the Golgi apparatus. Indeed, we found that expression of PDE defective mutant (SdeA H277A) or mART defective mutant (SdeA EE/AA) did not exhibit significant impact on the structure of the Golgi (Fig. 2A, B). In addition, the effect of wild-type SdeA on the Golgi structure could be counteracted by expression of DupA, but not its catalytically mutant DupA H67A (Fig. 2A, B). These findings suggest that Golgi disruption observed in cells expressing SdeA is likely caused by the accumulation of ubiquitinated substrates. These observations are in agreement with previous study [19]. Similar result was also obtained in HeLa cells stained with both cis (GM130) and trans
(TGN46) Golgi marker antibodies (Fig. S1B). In order to evaluate the physiological relevance of SdeA in triggering Golgi disruption, we infected human lung carcinoma A549 cells with either a wildtype Legionella strain, or mutant strains ( $\triangle$ sidEs or $\triangle d u p A / B$ ). As expected, we observed a scattering of the Golgi apparatus in cells infected with wild-type but not $\triangle$ sidEs Legionella or control cells. Infection by Legionella without DupA/B caused more dramatic dispersal of the Golgi, compared to the wild-type Legionella (Fig. 2C, D). To further dissect the modulation of Golgi by SdeA, we analyzed the Golgi morphology of the cells expressing SdeA with super-resolution microscopy. Data from DNA-PAINT super-resolution microscopy shows that the Golgi structure was damaged, however cis Golgi protein GM130 and trans Golgi protein Golgin97 were still colocalized in cells expressing SdeA (Fig. S1C). Moreover, SdeA expression did not change the level of the proteins required for Golgi structure maintenance (Fig. S1D). Taken all together, these data suggest that SdeA mediated PRubiquitination of host substrates induces disruption but not complete fragmentation of Golgi ribbon.

\section{In vitro and in vivo validation of PR-ubiquitination of Golgi substrates by SdeA}

Using the PR-deubiquitinase DupA as a bait, we pulled-down over 180 potential host substrate proteins of SdeA [15]. Notably, Golgi proteins GRASP55 (gene name: GORASP2) and GCP60 (gene name: $A C B D 3$ ) had the highest ratios among these putative Golgi protein substrates (Fig. 3A). Since GRASP55 plays important roles in the maintenance of the Golgi structure $[20,21]$, we hypothesized that SdeA modifies and inactivates Golgi proteins related to structure maintenance, thereby inducing Golgi disruption. GRASP65, which 
A

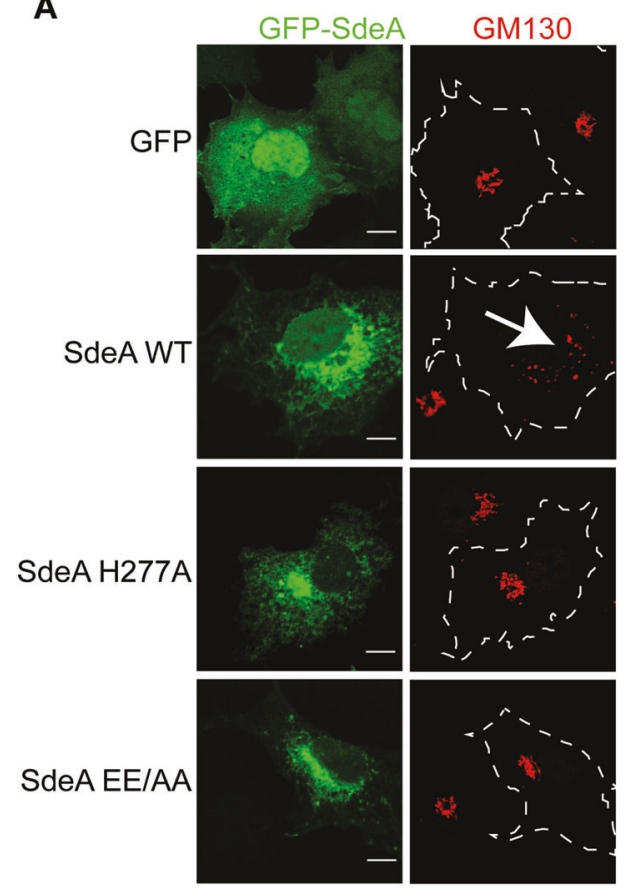

C
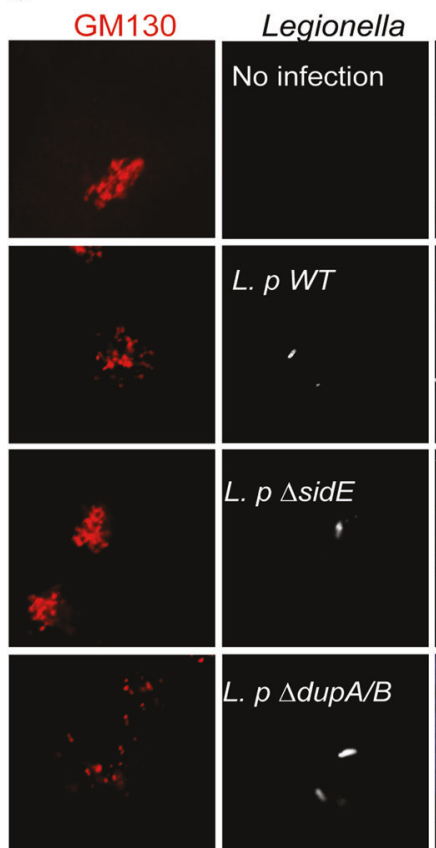

Merge
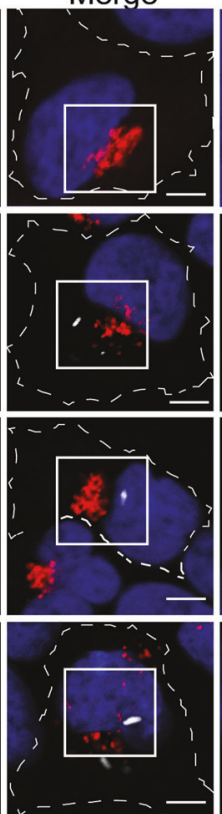
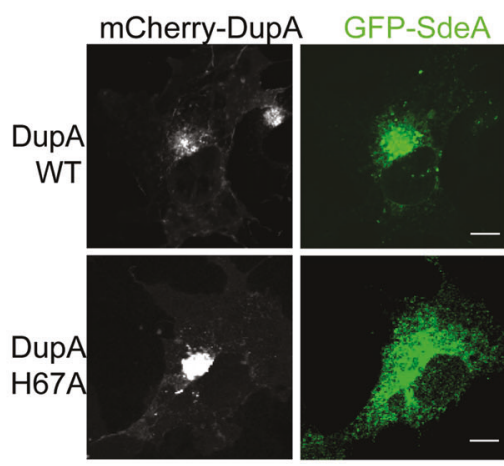

GM130

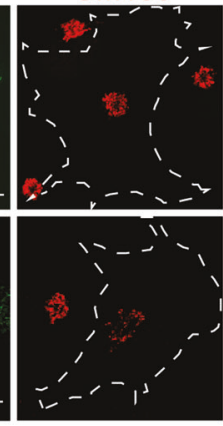

B

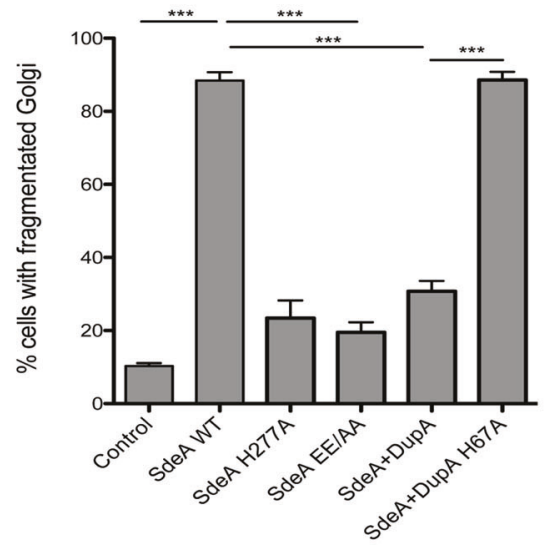

Inset
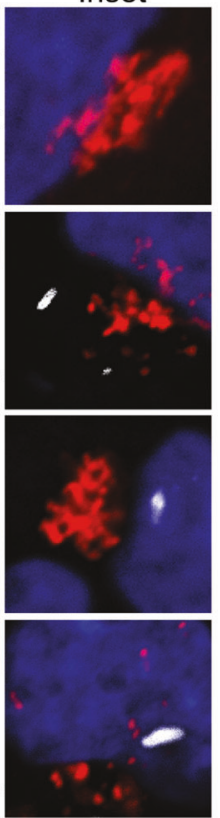

D

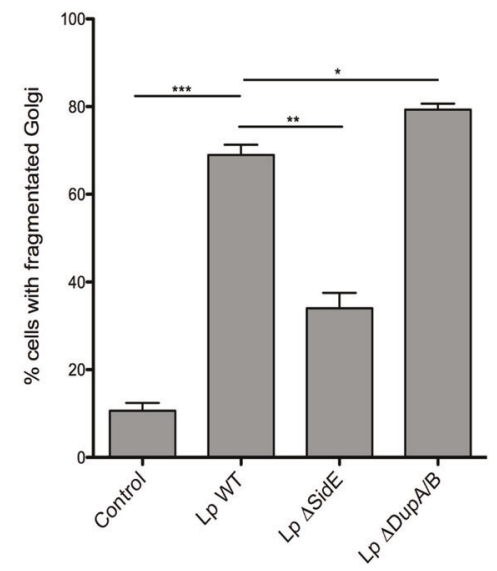

Fig. 2 Legionella effector SdeA mediates Golgi fragmentation in cells. A Confocal images showing Golgi (red) fragmentation caused by exogenously expressed SdeA (green). GFP-tagged SdeA wild-type or catalytically defective mutants were expressed or co-expressed with DupA in COS7 cells. Cells were cultured for $24 \mathrm{~h}$ after transfection then fixed with $4 \%$ PFA. Scale bars, $10 \mu \mathrm{m}$. B Quantification of the percentage of cells with dispersed Golgi in (A). Data are shown as means \pm SEM of more than 60 cells taken from three independent experiments. ${ }^{* * *} P<0.001$. C Confocal images showing Golgi fragmentation caused by Legionella. A549 cells were infected with wild-type or mutant Legionella as indicated. Cells were washed 3 times with PBS after $2 \mathrm{~h}$ infection to remove non-phagocytosed bacteria, then fixed with 4\% PFA and stained with indicated antibodies. Scale bars, $5 \mu \mathrm{m}$. D Quantification of the percentage of cells with dispersed Golgi in (C) Data are shown as means \pm SEM of more than 70 cells taken from three independent experiments. Data were analyzed with unpaired $t$ test, ${ }^{* * *} P<0.001,{ }^{*} P<0.01,{ }^{*} P<0.05$. 
A

\begin{tabular}{lll}
\hline & Protein names & dDup12/dSidE \\
\hline & STIM1 & 5,73 \\
& FAM134C & 4,86 \\
ER & VCP & 4,59 \\
proteins & RTN4 & 4,41 \\
& SNAP23 & 4,29 \\
& TEX264 & 3,64 \\
& LNP & 2,38 \\
& & \\
& GRASP55 & 5,83 \\
Golgi & TMED8 & 4,72 \\
related & GCP60 & 2,69 \\
proteins & YIF1A & 2,44 \\
& RAB33B & 3,09 \\
& SNX5 & 2,76 \\
\hline
\end{tabular}

C
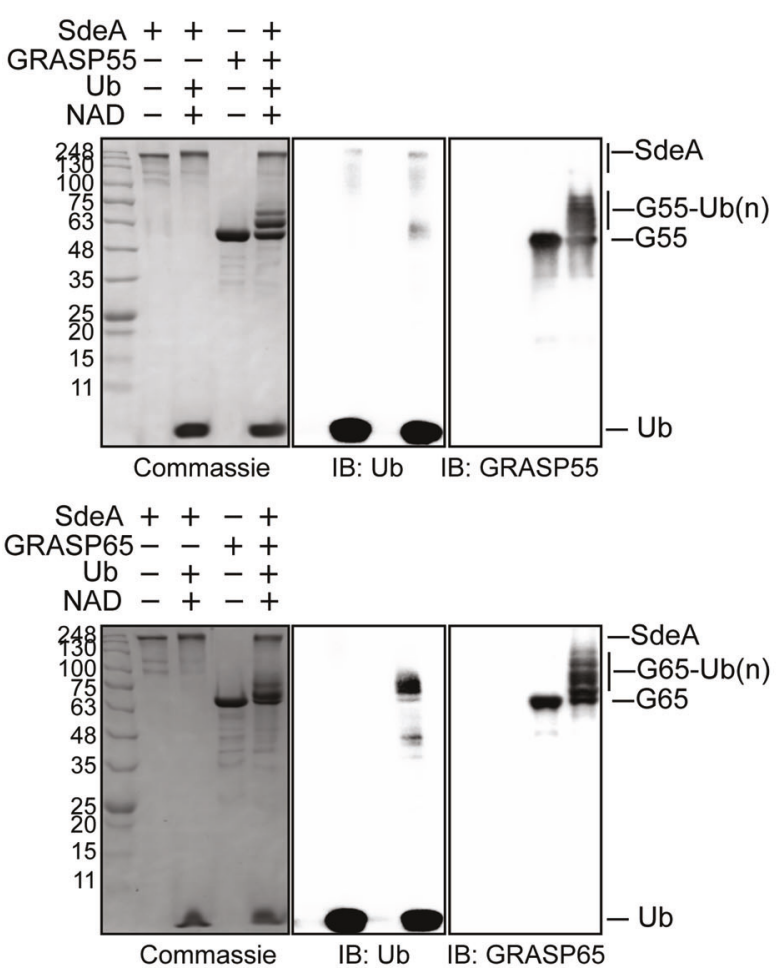

B

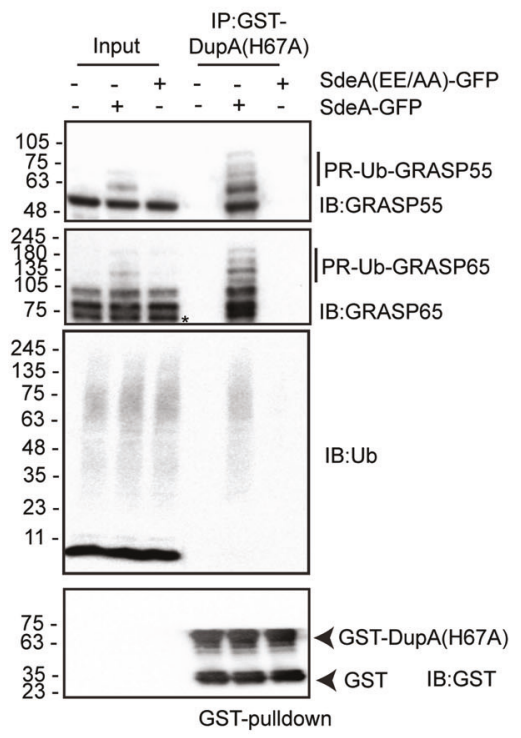

D
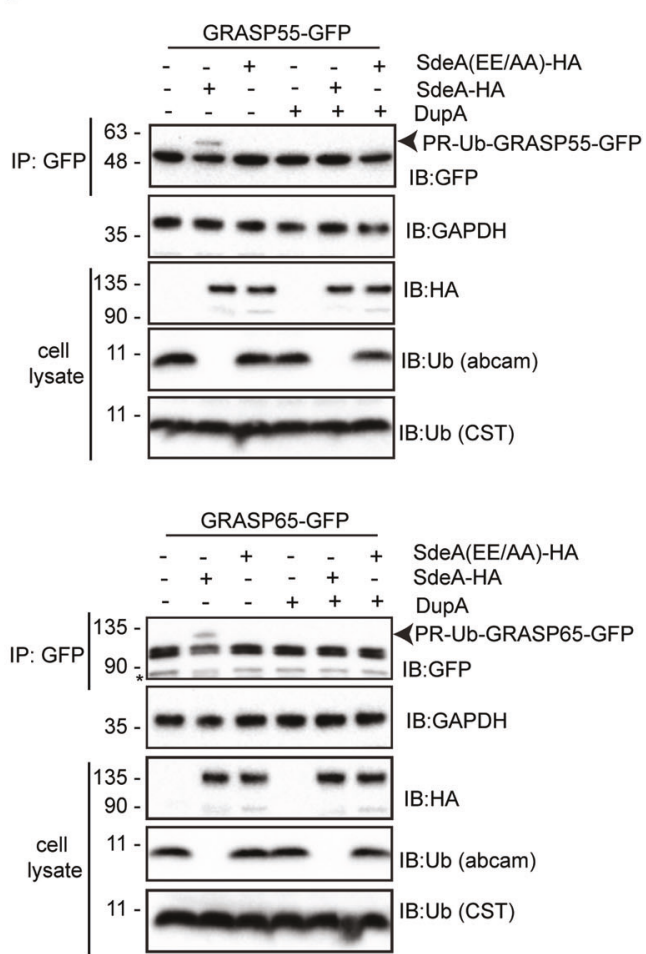

Fig. 3 SdeA ubiquitinates Golgi tethering factor GRASP proteins. A Potential ER and Golgi protein substrates of SdeA identified by mass spectrometry. Values indicate intensity ratios between proteins enriched from samples infected with different Legionella strains $(\triangle d u p A / B$ over $\triangle$ sidE). Among the substrate candidates, Golgi tethering factor GRASP55 (red) is one of the highly ubiquitinated proteins. B Modification of endogenous GRASP55 and GRASP65 by SdeA. Cell lysates from HEK293T cells expressing SdeA or SdeA EE/AA were incubated with GST-DupA (H67A) trapping mutant and blotted for GRASP55 and GRASP65. C GRASP55 and GRASP65 ubiquitination by SdeA in vitro. Purified GRASP55 or GRASP65 were incubated with SdeA in the presence of NAD ${ }^{+}$and ubiquitin. Reaction products were separated with SDS-PAGE and then stained with Coomassie blue or blotted with antibodies against ubiquitin, GRASP55 or GRASP65. D Modification of overexpressed GRASP55 or GRASP65 by SdeA in cells. HEK293T cells were co-transfected with GFP-tagged GRASP55 or GRASP65 and wild type SdeA or indicated SdeA mutants, after $24 \mathrm{~h}$ cells were collected and lysed, GFP tagged proteins were isolated with GFP-trap beads, treated with purified DupA and separated with SDS-PAGE followed by blotting with antibody against GFP. For total cell lysate, the cell signaling technology (CST) antibody against ubiquitin was used to detect total ubiquitin, abcam antibody that does not detect ubiquitin modified by SdeA was used to indicate the expression and activity SdeA.

shares high sequence similarity with GRASP55, is localized to the cis Golgi and is also found in dispersed Golgi apparatus in cells expressing wild-type SdeA (Fig. S2A). To test this hypothesis, firstly we incubated GST-DupA H67A trapping mutant with cell lysate from cells expressing SdeA and blotted the pulldown product with antibodies against GRASP55 and GRASP65 to validate if SdeA modifies endogenous GRASP proteins. The blots show that SdeA expression indeed caused PR-ubiquitination of both GRASP55 and GRASP65 (Fig. 3B). In vitro ubiquitination assays were performed to further confirm the potential PR-ubiquitination of these two 
A

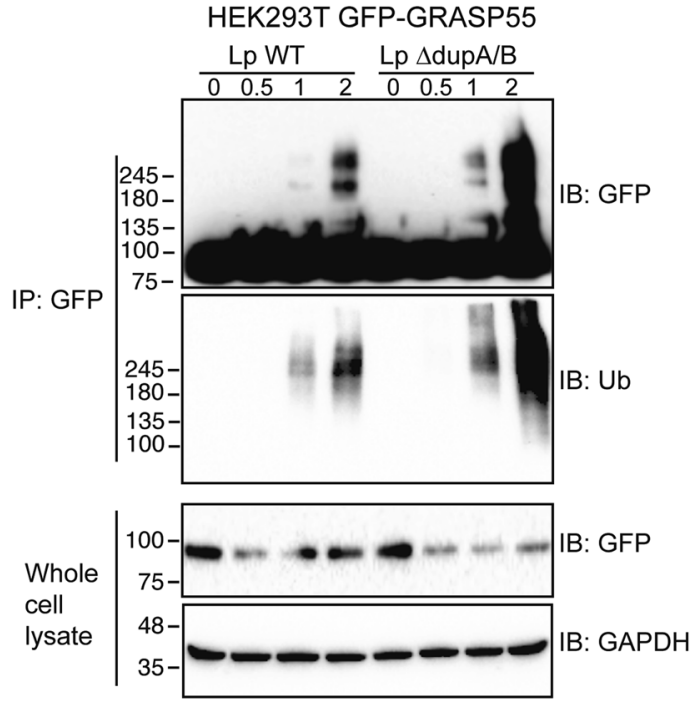

C

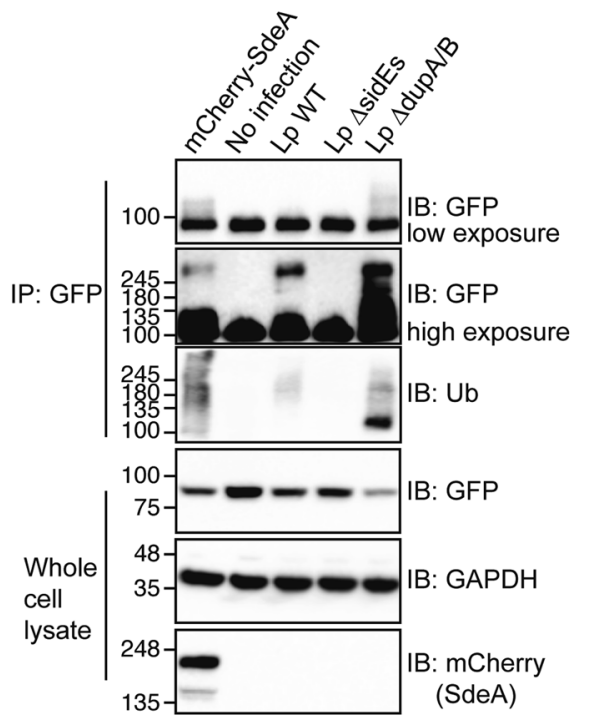

B
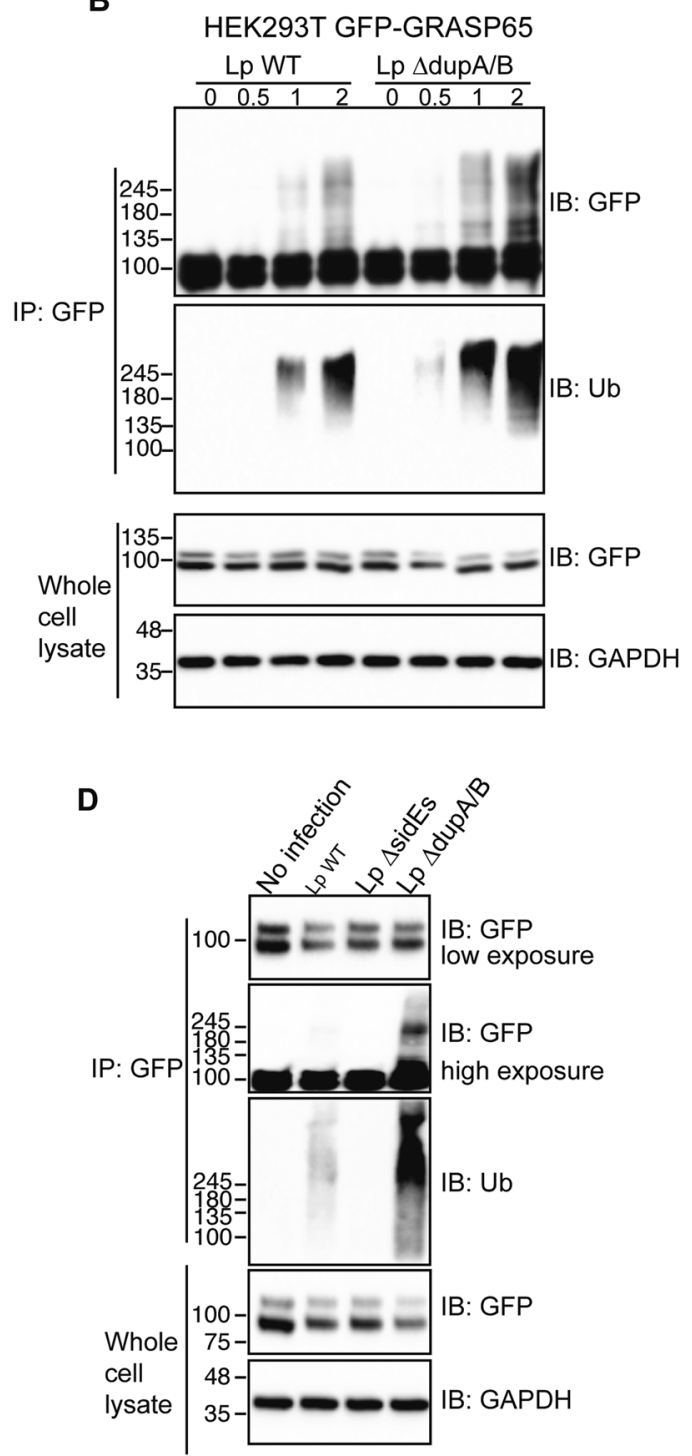

Fig. 4 Legionella infection causes ubiquitination of GRASP proteins, which is dependent on SidE effectors. A Ubiquitination assay of GRASP55-GFP purified from HEK293T cells infected with Legionella strains. HEK293T cells were seeded in 6-well plate and co-transfected with plasmids encoding C-terminally GFP tagged GRASP55 and Fc $\gamma$ Rll then were infected for indicated times with Legionella bacteria opsonized by Legionella antibody. Cells were lysed with IP lysis buffer and purified GRASP55 proteins were separated by SDS-PAGE followed by blotting using anti-GFP and anti-ubiquitin antibodies. B Ubiquitination assay of GRASP65-GFP purified from HEK293T cells infected by Legionella wild type or $\triangle d u p A / B$ mutant. C Ubiquitination assay of GRASP55-GFP purified from HEK293T cells infected with Legionella wild-type, $\Delta$ sidEs or $\triangle d u p A / B$ strains. D Ubiquitination assay of GRASP65-GFP purified from HEK293T cells infected with Legionella wild-type, $\Delta$ sidEs or $\Delta d u p A / B$ strains.

Golgi proteins by SdeA. We observed that SdeA is also able to modify both GRASP55 and GRASP65 in vitro (Fig. 3C). Furthermore, cellular expression of wild-type SdeA, but not inactive mART mutant, resulted in the appearance of ubiquitinated GRASP55 and GRASP65 in cells. This PR-ubiquitination was lost when pure DupA was added to immunoprecipitated GFP-tagged GRASP55 or GRASP65 in vitro (Fig. 3D). Similar observations were made for GCP60, where purified GCP60 from cells incubated with wild-type SdeA exhibited PR-ubiquitination (Fig. S2B). Such modification also appeared in cells when GCP60 was co-expressed with wild-type SdeA but not upon co-expression of SdeA EE/AA mutant (Fig. S2C). Along our hypothesis that SdeA is actively targeted to the Golgi, exogenous expression of CT-truncated SdeA mutants showed markedly reduced activity in modifying substrate GRASP55 (Fig. S2D), similar to the effect observed on PR-ubiquitination of Rab33b, indicating that Golgi localization of SdeA is important for substrate modification. Together, these results suggest that the PR-ubiquitination of Golgi tethering proteins GRASP55, GRASP65 and GCP60 by SdeA is a selective and functional part of the hijacking strategy of Legionella.

\section{Legionella infection causes PR-ubiquitination of GRASP55 and GRASP65}

To check whether these Golgi proteins are PR-ubiquitinated upon Legionella infection, we immunoprecipitated GFP-tagged GRASP55 and GRASP65 from HEK293T cells infected with Legionella strains and analyzed them for PR-ubiquitination. The results showed that both GRASP55 and GRASP65 were ubiquitinated in a timedepending manner following Legionella infection (Fig. 4A, B). Legionella infection-induced GRASP55 and GRASP65 PRubiquitination was lost when cells were infected with Legionella $\triangle$ sidEs, and was increased in cells infected with the Legionella 
A

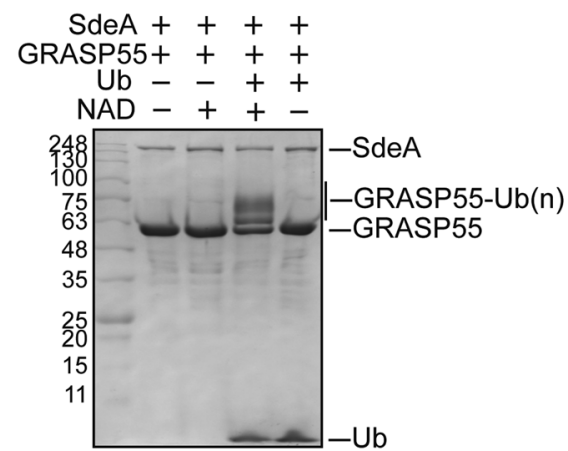

C

mCherry-SdeA EE/AA WT WT GRASP55-HA WT WT $7 S^{*}$

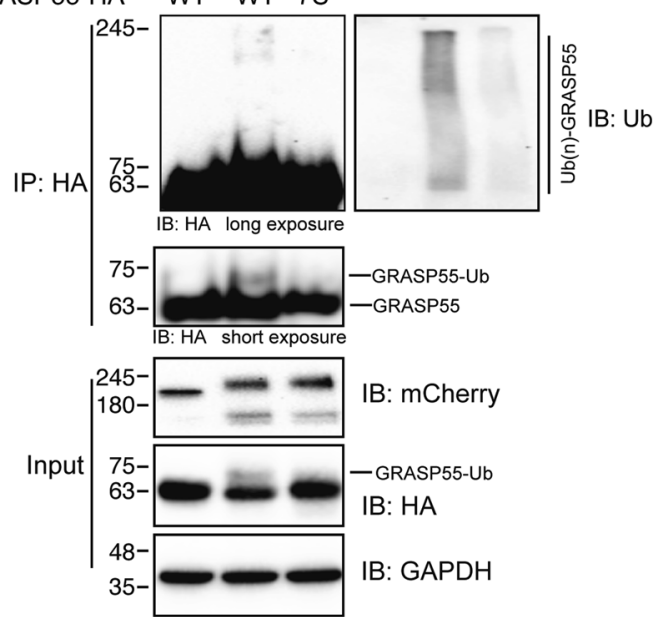

B

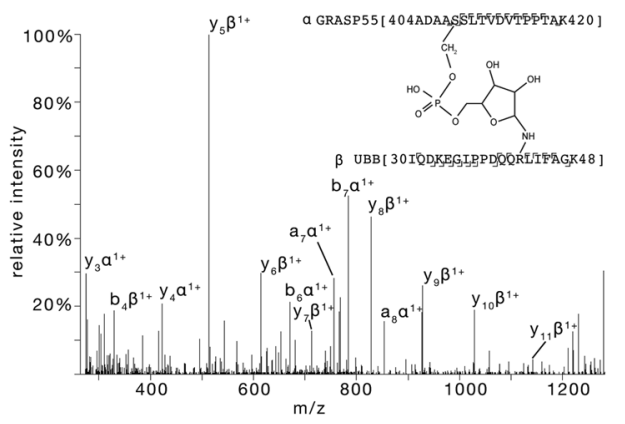

D

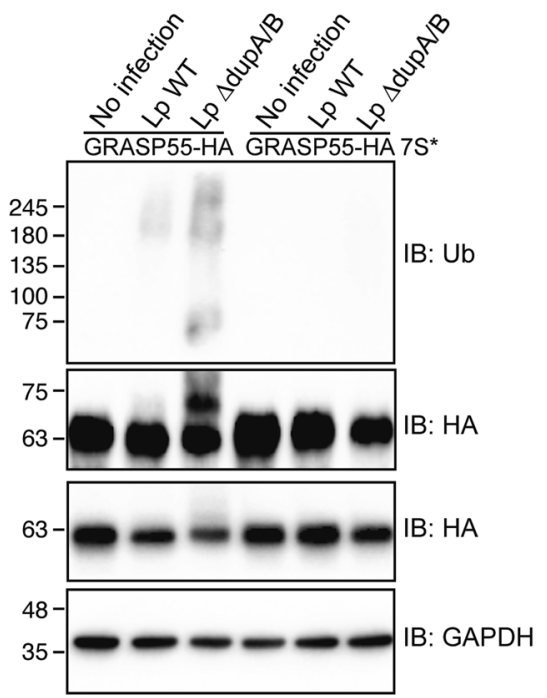

Fig. 5 Identification of GRASP55 ubiquitination with mass spectrometry. A In vitro reaction of GRASP55 ubiquitination by SdeA for mass spectrometry analyses. In total, $20 \mu \mathrm{g}$ purified GRASP55 was incubated with SdeA and ubiquitin in the present of NAD. 10\% reaction products were separated with SDS-PAGE and then stained with Coomassie blue or blotted with with antibodies against ubiquitin, GRASP55 to check the ubiquitination, the rest samples were subjected to mass spectrometry analyses. B Spectrum of GRASP S408-ubiquitin cross-linked peptide. C Validation of of ubiquitination sites in GRASP55. C-terminally HA-tagged wild-type and GRASP55 mutant were co-expressed with SdeA in HEK293T cells. After $24 \mathrm{~h}$ the cells were lysed for HA immunoprecipitation. Purified GRASP55-HA proteins were separated with SDS-PAGE followed by blotting using anti-HA and anti-ubiquitin antibodies. D Ubiquitination assay of wild type GRASP55 and mutant in cells infected with Legionella.

$\triangle d u p A / B$ (Fig. 4C, D), thus, confirming that SidE family effectors are essential for PR-ubiquitination of host substrate proteins. Similar results were obtained for GCP60 (Fig. S3A). Moreover, DupA was able to remove the ubiquitination of GRASP55 and GRASP65 induced by Legionella infection (Fig. S3B, C). These data suggest that SdeA PR-ubiquitinates Golgi tethering proteins GRASP55 and GRASP65 during Legionella infection, further supporting our hypothesis that this modification has a directed function.

\section{SdeA ubiquitinates multiple serines of GRASP55 protein}

Previous studies provided insights how SdeA targets and bridges Arg42 of Ub to serine residues of substrate proteins via a phosphoribosyl linker $[9,10]$. To gain insight into the mechanism of activity regulation of GRASP proteins by PR-ubiquitination, we used mass spectrometry to identify modified residues on GRASP55 following in vitro ubiquitination by SdeA (Fig. 5A). Four modified serine residues were identified in GRASP55 (S3, S408, S409, S449) (Fig. 5B, Fig. S4). To further confirm these ubiquitination sites, we replaced seven serine residues (GRASP55 $7 \mathrm{~S}^{*}$ ), including the identified serines and their adjacent serines, by either threonine (S3, S4, S449, S451) or alanine (S408, S409, S441). We observed that ubiquitination of GRASP55 in cells co-expressing SdeA was markedly decreased when the serines were replaced (Fig. 5C). Similarly, we confirmed that GRASP55 bearing the seven mutated serine residues can not be ubiquitinated when cells were infected with wild-type or $\triangle d u p A / B$ Legionella strains (Fig. 5D). These results confirm that these identified serines are the primary targets.

\section{PR-ubiquitination disrupts GRASP interactions}

Studies have shown that GRASP proteins function in the connection of Golgi stacks and thereby Golgi structure maintenance through self-interaction and interactions with Golgi matrix proteins $[20,22,23]$. Their activity can be regulated by post-translational modifications, for example, phosphorylation of serines within GRASP proteins was shown to result in Golgi fragmentation [24]. We hypothesized that PR-ubiquitination of serines in GRASP proteins may affect self-interactions that are necessary for the connection of the Golgi stacks. To test this, we firstly PR-ubiquitinated purified GRASP55-GFP in vitro and then incubated the modified GRASP55 with purified His-tagged GRASP55. Co-IP analyses showed that PR-ubiquitinated GRASP55 exhibited reduced self-interaction compared to unmodified GRASP55 (Fig. 6A). This effect could also be seen in cells when the HA-tagged wild type GRASP55 or GRASP55 $7 \mathrm{~S}^{*}$ serine mutant were co-expressed with GFP-tagged GRASP55 $7 \mathrm{~S}^{*}$ in the presence of SdeA. The capacity of PR-ubiquitinated wild-type HA-GRASP55 to self-interact with GFP-GRASP55 $7 \mathrm{~S}^{*}$ was decreased in comparison to SdeA resistant HA-GRASP55 $7 \mathrm{~S}^{*}$ (Fig. 6B). To 
A

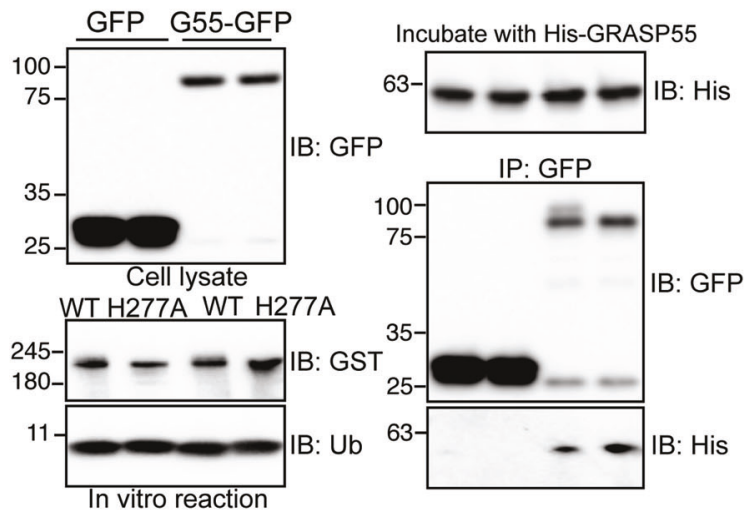

B

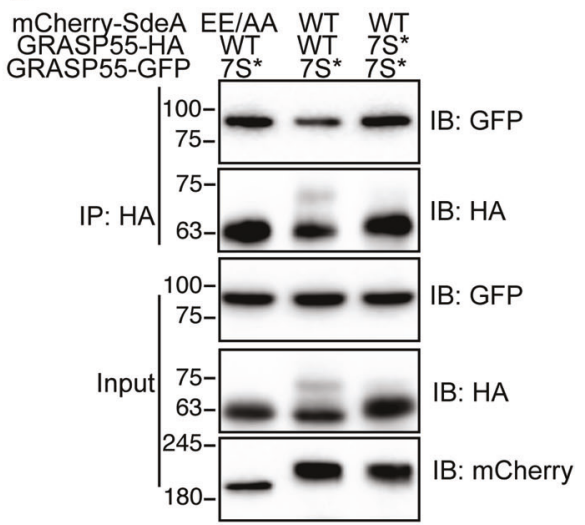

C
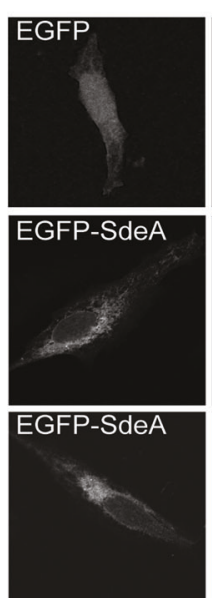

HA

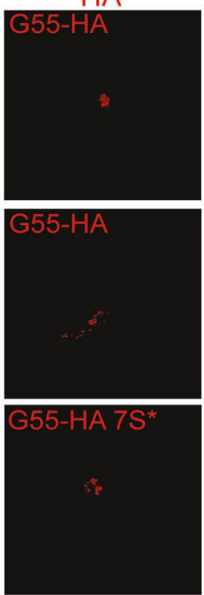

GM130

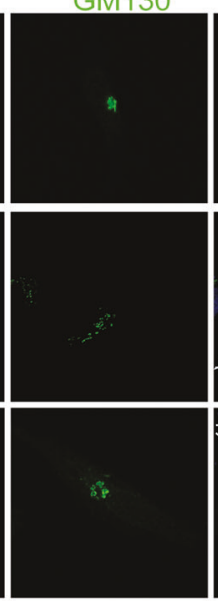

Merge

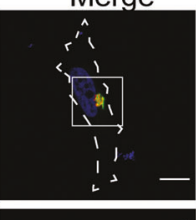

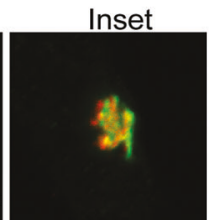

D

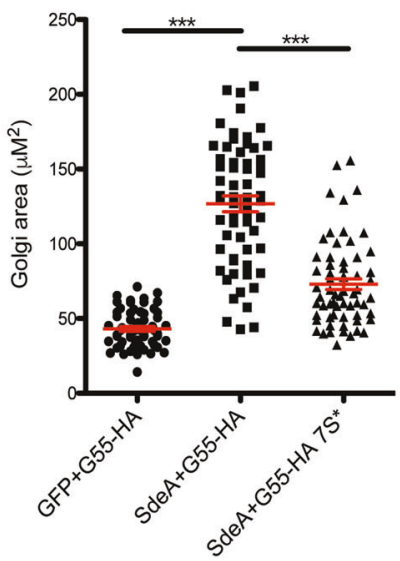

Fig. 6 Serine ubiquitination impairs GRASP55 function. A Assay of the effect of PR-ubiquitination on GRASP55 dimerization in vitro. GRASP55-GFP purified from HEK293T cells were modified in vitro using SdeA and ubiquitin in the presence of NAD ${ }^{+}$, ubiquitinated GRASP55GFP was then incubated with purified His-tagged GRASP55. Interaction between differently tagged GRASP55 proteins were analyzed with coimmunoprecipitation followed with western blotting. B Assay of the effect of PR-ubiquitination on GRASP55 dimerization in vivo. HA-tagged GRASP55 and GFP-tagged GRASP55 serine mutant were co-expressed with SdeA in HEK293T cells. Protein interaction between differently tagged GRASP55 were analyzed with co-IP and western blotting. C Confocal images showing that GRASP55 mutant is resistant to Golgi fragmentation caused by SdeA expression. Golgi areas of more than 60 cells from 3 replicates of each condition were measured with ImageJ software. Scale bars, $10 \mu \mathrm{m}$. D Data are shown as means \pm SEM of more than 70 cells taken from three independent experiments. Data were analyzed with unpaired $t$ test, ${ }^{* * *} P<0.001$.

analyze the functional impact of this observation on cells, we expressed wild-type GRASP55 or the GRASP55 $7 \mathrm{~S}^{*}$ serine mutant in GRASP55/GRASP65 KO HeLa cells, then monitored the structural stability of the Golgi in cells co-expressing SdeA. As previously shown, double knockout of GRASP55 and GRASP65 induced dispersal of the Golgi [25] (Fig. S5). This phenotype could be rescued by ectopic expression of either wild-type GRASP55 or GRASP55 $7 \mathrm{~S}^{*}$ (Fig. S5), suggesting that serine mutations do not interfere with the function of GRASP55. Golgi disruption reoccurred when SdeA was concomitantly expressed with GRASP55 (Fig. 6C, D). However, the Golgi apparatus appeared less scattered when GRASP55 $7 \mathrm{~S}^{*}$ was expressed, indicating that the higher resistance of GRASP55 serine mutant to SdeA ubiquitination activity results in increased structural stability of the Golgi in cells expressing SdeA (Fig. 6C, D). This data indicates that SdeA-caused Golgi disruption is supposedly the result of the modification of GRASP proteins, disturbing the connection between Golgi stacks.

Legionella containing vacuole does not recruit Golgi scatters Intracellular pathogens tend to create a membrane surrounded niche for maturation, proliferation, and escape from defense mechanisms such as selective autophagy within the host cell.
Along this line, Chlamydia infection induces Golgi fragmentation in order to generate Golgi ministacks for bacterial inclusions [26]. As for Legionella, Legionella containing vacuoles (LCVs) recruit ER membranes, thus converting the phagosome into a specific compartment that has features of ER $[15,17,27]$. We hypothesized that Legionella infection induces Golgi dispersal in order to facilitate the fusion of vesicles from the Golgi with LCV to enhance the formation of LCV and, ultimately, intracellular replication. To test this hypothesis, we infected HEK293T cells overexpressing GRASP55 or trans-Golgi marker GalT. The immunostaining showed that exogenous GRASP55 was recruited to LCV, however, our study recognized the fact that exogenously overexpressed GRASP55 and GalT were shown to be partially localized in ER, which can be remodeled and recruited to LCV during infection. The recruited GRASP55 could very well be derived from the ER, and not the fragmented Golgi (Fig. 7A, B). To further address whether Legionella recruits fragmented Golgi cargo, we infected A549 cells with Legionella, stained cells with antibodies against endogenous cis-Golgi protein GM130 or trans-Golgi protein TGN46. Immunostaining results suggested that neither cis-Golgi marker nor trans-Golgi accumulated on LCV (Fig. 7C, D). These data suggest that against our initial hypothesis Legionella does not 
A
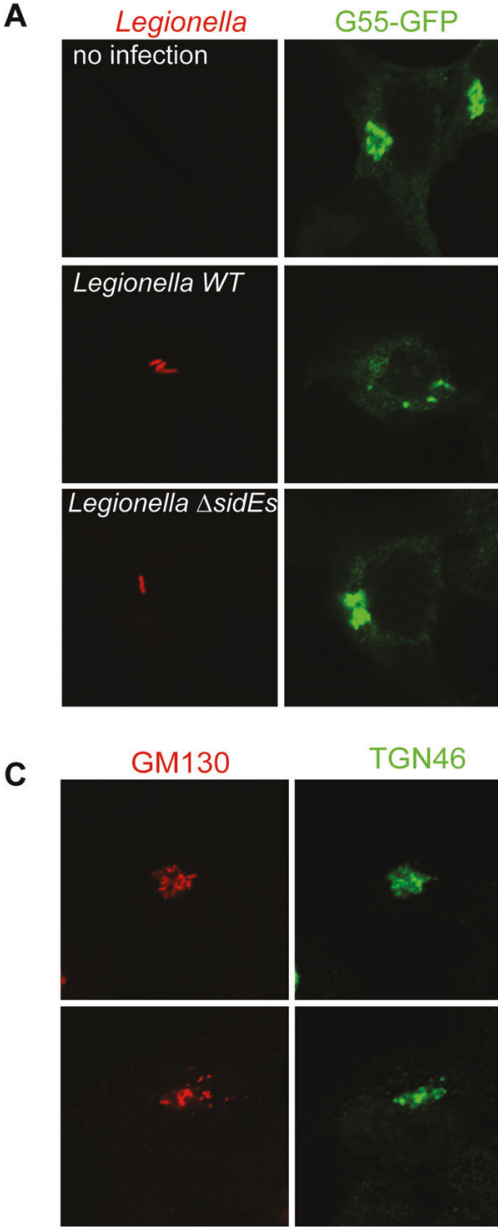

D

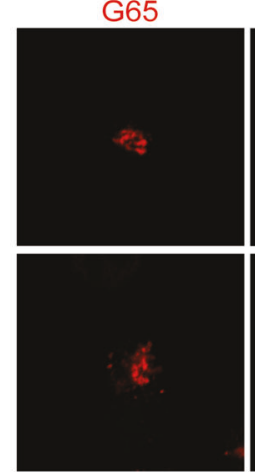

G55
Merge
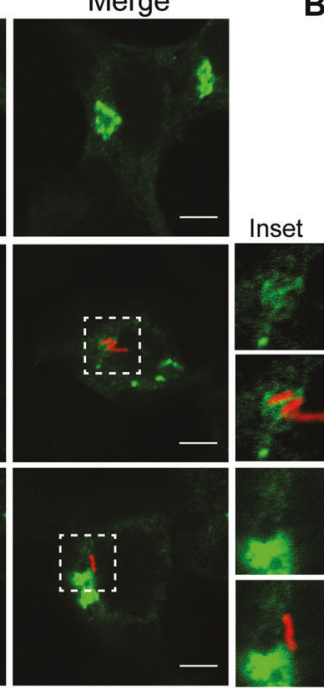

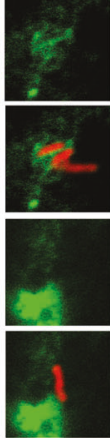

B
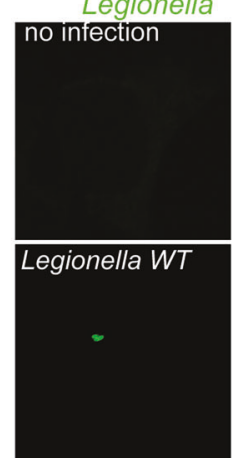

Legionella $\Delta$ sidEs

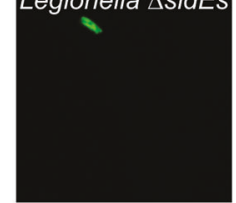

mCherry-GalT
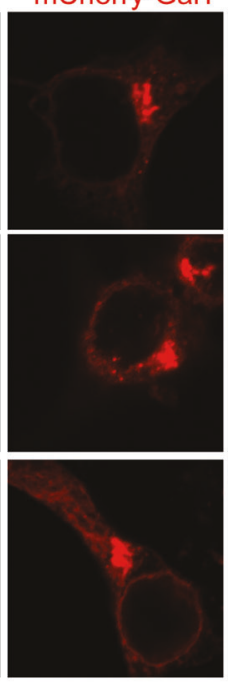

Inset
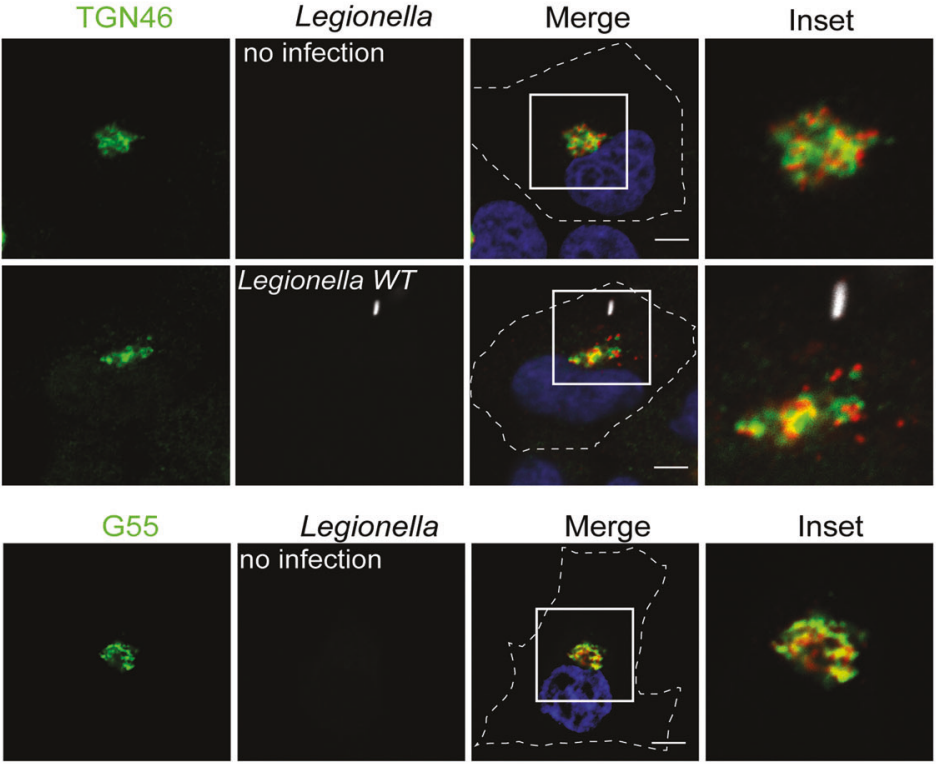

Legionella
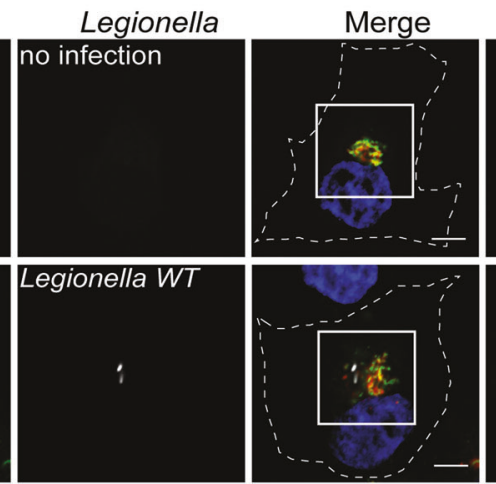

Inset

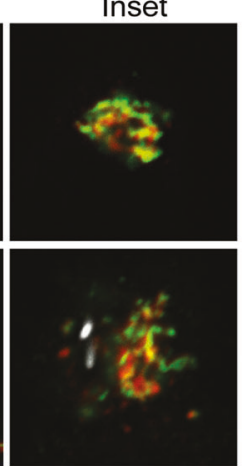

Fig. 7 Legionella does not recruit fragmented Golgi. A Confocal images showing Legionella recruits overexpressed Golgi protein GRASP55. HEK293T cells transfected with plasmids encoding FC $\gamma$ RII and GFP-tagged GRASP55 were infected with indicated Legionella strains. Cells were washed 3 times with PBS after $2 \mathrm{~h}$ infection to remove un-endocytosed bacteria, then fixed with $4 \%$ PFA and stained with antibody against Legionella. Scale bars, $5 \mu \mathrm{m}$. B Confocal images showing Legionella recruits overexpressed Golgi marker GalT. Scale bars, $5 \mu \mathrm{m}$. C Confocal images showing Legionella does not recruit endogenous cis-Golgi protein GM130 or trans-Golgi protein TGN46. A549 cells were infected with Legionella expressing dsRed and stained with antibodies against GM130 and TGN46. Scale bars, $5 \mu \mathrm{m}$. D Confocal images showing Legionella does not recruit endogenous cis-Golgi protein GRASP65 or trans-Golgi protein GRASP55. A549 cells were infected with Legionella expressing dsRed and stained with antibodies against GRASP65 and GRASP55. Scale bars, $5 \mu \mathrm{m}$.

disperse the Golgi simply to recruit Golgi-derived vesicles for the creation of LCVs, but that there must be another functional reasoning behind.

\section{Serine ubiquitination regulates secretory pathway in host cells}

In eukaryotic cells, the Golgi stack receives newly synthesized proteins from the ER, proteins then undergo modifications before being sorted via the trans-Golgi network. To investigate the effect of SdeA mediated Golgi disruption on Golgi function, we performed fluorescence recovery after photobleaching (FRAP) assay, the data indicates that the recovery of fluorescence after photobleaching of marked region is slower in SdeA expressing cells (Fig. S6). Based on this observation, we then asked that whether SdeA inhibits protein trafficking via the Golgi. Vesicular stomatitis virus glycoprotein (VSVG) is a transmembrane protein that has been widely used as a tool to monitor protein trafficking through the secretory pathway $[28,29]$. To access the functionality 
A
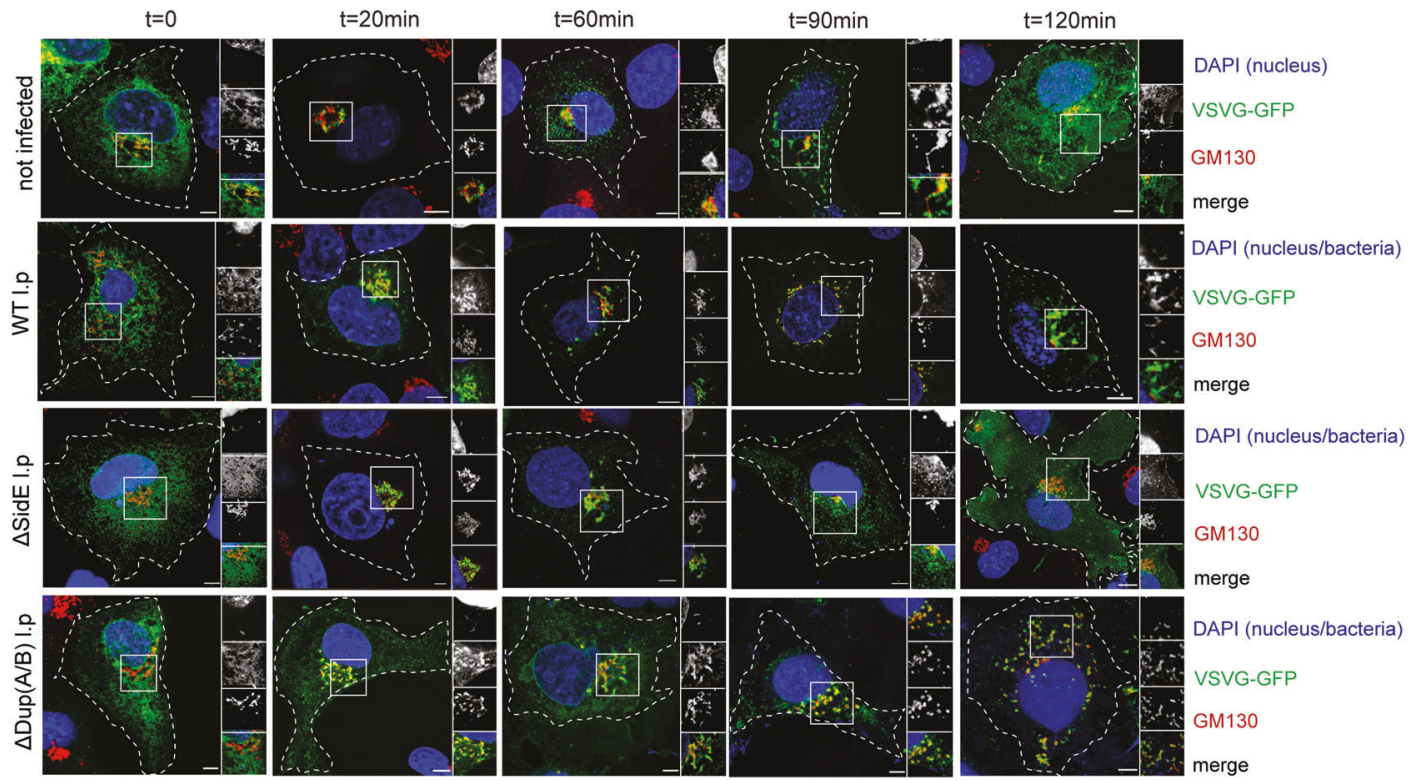

B

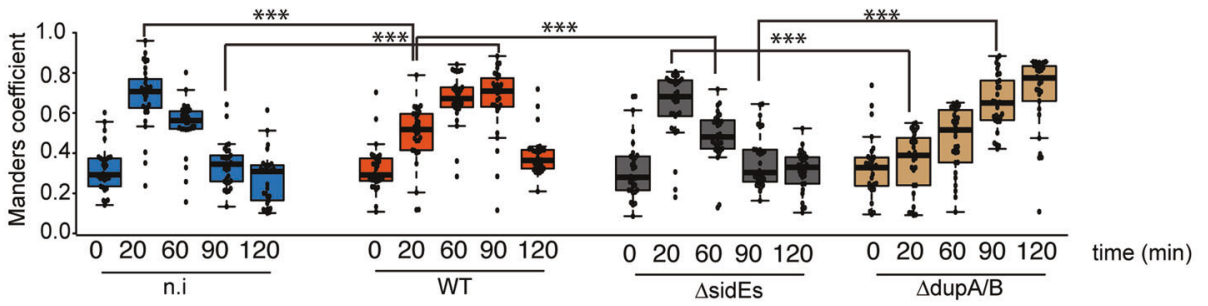

C
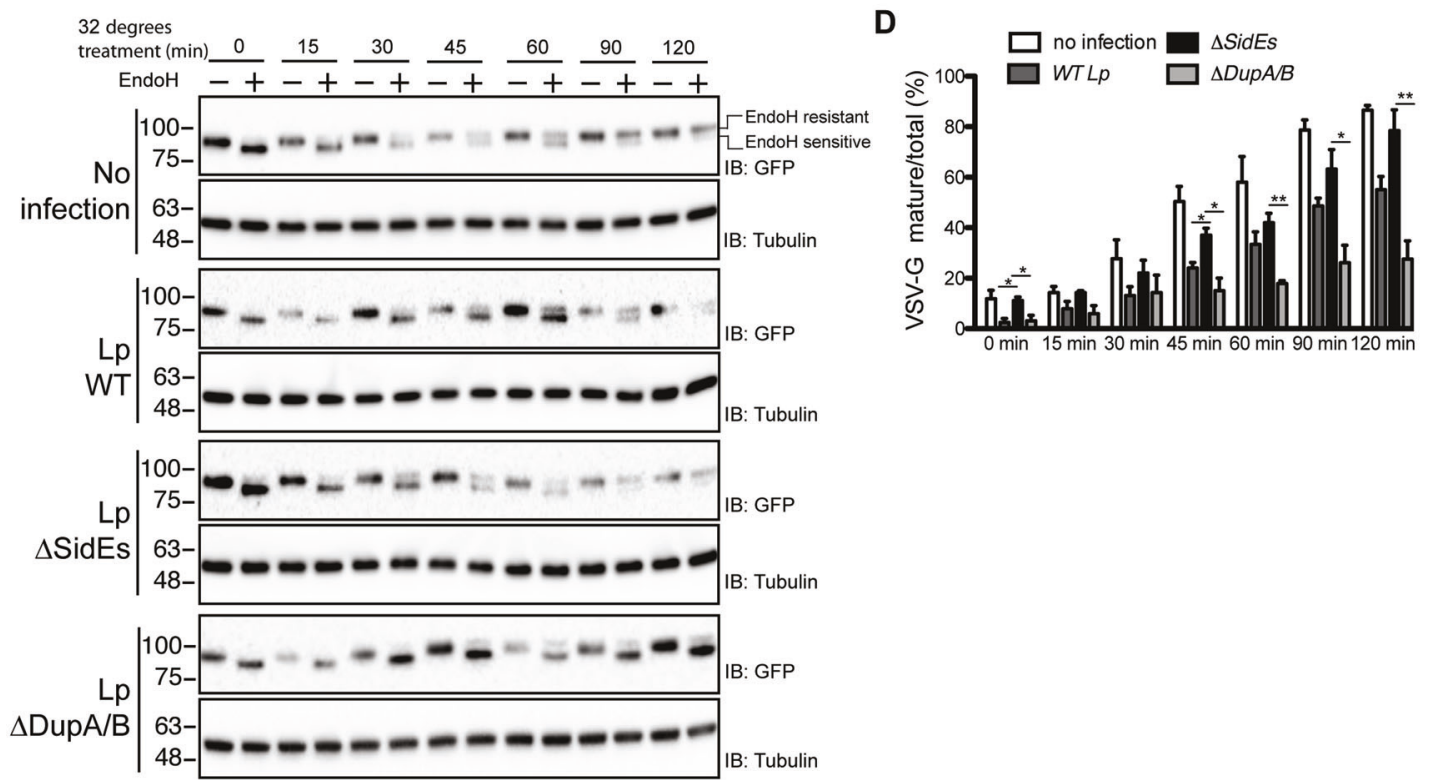

Fig. 8 SdeA induced serine ubiquitination inhibits VSVG trafficking through Golgi membranes. A Confocal images showing the effect of SidE family effectors on VSVG trafficking during Legionella infection. A549 cells were transfected with VSV-G-GFP and cultured at $37^{\circ} \mathrm{C}$ for $24 \mathrm{~h}$ to express the proteins before transferred to $40^{\circ} \mathrm{C}$. After $16 \mathrm{~h}$ incubation at $40^{\circ} \mathrm{C}$, cells were infected with Legionella for another $2 \mathrm{~h}$ then washed 3 times with PBS then moved to $32^{\circ} \mathrm{C}$ for $0 \mathrm{~min}, 10 \mathrm{~min}, 20 \mathrm{~min}, 60 \mathrm{~min}, 90 \mathrm{~min}, 120 \mathrm{~min}$ to release VSVG from ER. A549 cells were fixed and VSVG trafficking was acquired with confocal microscopy after immunofluorescence staining. Scale bars, $5 \mu \mathrm{m}$. B Quantitative analysis of the effect of SidE family effectors on VSVG trafficking during Legionella infection. Colocalization between VSVG and GM130 was shown as Manders coefficient. Data represents 30 cells taken from 3 independent experiments. White boxes indicate insets which are split into red, green, blue channels and displayed on the right side of the image. Center lines show the medians; box limits indicate the 25th and 75th percentiles as determined by $\mathrm{R}$ software; whiskers extend 1.5 times the interquartile range from the 25th and 75th percentiles, outliers are represented by dots; data points are plotted as circles. C Western blotting analysis of the effect of SidE family effectors on VSVG trafficking during Legionella infection using EndoH. Upper bands indicate the EndoH resistant form (mature) and lower bands indicate the EndoH sensitive form of VSVG. D Quantification of $(\mathbf{C})$ to indicate the effect of Legionella infection on the conversion of EndoH sensitive form to resistant form of VSVG upon $32{ }^{\circ} \mathrm{C}$ incubation. Data were analyzed with unpaired $t$ test, ${ }^{* * *} P<0.001,{ }^{* *} P<0.01,{ }^{*} P<0.05$. 
of the Golgi apparatus upon Legionella infection, we used a VSVGGFP tracker protein to follow its transit through the Golgi. Immunofluorescence analyses indicated that in control A549 cells or cells infected with Legionella SidEs deletion strain, VSVG reached its peak of accumulation in the Golgi after 20 min of incubation at $32{ }^{\circ} \mathrm{C}$, and the colocalization index then gradually decreased as the protein is trafficked from the Golgi to secretory vesicles. This process was slower in cells infected with wild-type Legionella or $\triangle d u p A / B$ mutant strain, where maximal colocalization of VSVG with the GM130 occurred at a later time point and was more prolonged, indicating lower efficiency of protein trafficking through the secretory pathway (Fig. 8A, B). This was further confirmed by monitoring the sensitivity of VSVG glycosylation to Endoglycosidase $\mathrm{H}$ (EndoH). EndoH is an enzyme that removes mannose rich ER resident protein but not complex forms of $\mathrm{N}$-like oligosaccharides from glycoproteins that are present in the Golgi or post Golgi compartments, thus has been widely used to monitor protein trafficking through the Golgi [30, 31]. To specifically analyze the effect of PR-ubiquitination on VSVG trafficking, we infected HEK293T cells at $40^{\circ} \mathrm{C}$ and collected cells lysates at different time points after incubation at $32{ }^{\circ} \mathrm{C}$, before treating them with EndoH. Western blots showed that the band shift of VSVG from EndoH sensitive form to resistant form was inhibited in cells infected with wild type Legionella or Legionella DupA/B deletion strain, compared with that in control cells or cells infected with Legionella $\Delta$ sidE strain, indicating that VSVG trafficking through the Golgi was suppressed (Fig. 8C, D). These data further demonstrate that PR-ubiquitination caused by SidE effectors decelerates protein trafficking through the Golgi. This is also confirmed with a VSVG assay in cells expressing SdeA (Fig. S7A, B). Taken together, these data demonstrate that Golgi disruption caused by SidE effectors impairs protein secretory pathway.

\section{DISCUSSION}

To date, considerable effort has been focused on investigating the mechanism and substrates of novel PR-ubiquitination catalyzed by SidE family of Legionella effectors. However, the functional consequences of PR-ubiquitination in the regulation of cellular processes has been poorly understood. In this study, we investigated the modification of Golgi proteins catalyzed by SidE effectors and explored the consequences of PR-ubiquitination in regulating Golgi morphology and secretory pathway.

By conducting in vitro reactions and MS-based analyses, we identified several serine residues in GRASP55 potentially modified by SdeA. Notably, mutation of these serines did not completely abolish the ubiquitination signal from purified GRASP55, suggesting that alternative residues in GRASP55 could also be modified by SdeA. This finding is consistent to other known substrates like Rab33b, in which S154 has been identified as a ubiquitination site for SdeA, yet S154A mutation does not abrogate ubiquitination [10]. SdeA appears to modify substrate serine sites independent of specific structural motifs and serines in the flexible regions are prone to be modified as shown for Rab proteins [14].

GRASP proteins contain a conserved N-terminal GRASP domain that is used to localize the proteins to the Golgi as well as to tether other GRASP proteins through trans-dimerization, which can be regulated through phosphorylation of the C-terminal serine and proline rich (SPR) domain by mitotic kinases [24, 32]. Several serines in this C-terminal region of GRASP55 including S408, S409, S441, S449 identified to be PR-ubiquitinated here, have also been reported to be phosphorylated in previous studies [33, 34]. Phosphorylation mimics at these sites disrupt the homodimerization of GRASP, possibly through protein conformational changes $[34,35]$. Based on the findings from a study using in vivo GRASP55/ 66 depletion, Grond et al. proposed that, instead of stacking the
Golgi cisternae core, GRASP proteins function in linking of the rims of Golgi cisternae and the consequent connection of Golgi stacks [20]. The disruption of GRASP protein homodimerization by PRubiquitination at these sites may lead to disconnection of Golgi stacks. Of note, in our previous study, some proteins related to, such as AKAP12, EPB41, SLK, were identified as possible substrates of SdeA [15]. This may also affect the assembly of organelles including the Golgi. More studies will be needed in future to answer the question whether Legionella regulates cytoskeleton organization through SdeA mediated PR-ubiquitination of these cytoskeletal proteins.

Many pathogens have been characterized to require host organelles for their own intracellular survival. As for Legionella, numerous host proteins have been detected on the LCVs. Of note, $\mathrm{PI}(4) \mathrm{P}$ decoration on LCV, which functions to recruit bacterial effectors during infection, was shown to be derived directly from the Golgi body of host cells [36]. However, our data in this study suggest that Legionella effectors disperse the Golgi but are not involved in the recruitment of Golgi components. This is in agreement with earlier studies, in which LCVs were purified from infected host cells and analyzed using proteomics approach, but Golgi proteins were rarely identified [37, 38]. During our ongoing study and preparation of this manuscript, Wan and colleagues reported Golgi fragmentation upon Legionella infection and the PR-ubiquitination of GRASP55. In their study it was shown that GRASP55 was recruited to LCV upon Legionella infection [39]. However, it should be noted that, unlike the endogenous GRASP55 protein that mainly localizes to the Golgi apparatus, overexpressed GRASP55 in their study was detected as largely localized to ER that could be recruited to LCV. The recruited GRASP55 could very well be derived from the ER, but not the dispersed Golgi. Recently, a study reported that PI(4)P-containing vesicles derived from Golgi are involved in mitochondria division [40]. Given the fact that mitochondrial dynamics is tightly modulated during Legionella infection [41], it is possible that Legionella SdeA affects mitochondria fission to facilitate bacterial replication. Further efforts will be needed to address the effect of PR-ubiquitination mediated Golgi disruption on mitochondria.

We have shown that PR-ubiquitination decelerates VSVG trafficking through the Golgi using microscopy and EndoH digestion assay. This finding is consistent with previous study showing that SdeA expression inhibits secretion of secreted embryonic alkaline phosphatase reporter (SEAP) [9].

Notably, multiple Legionella effectors have been suggested to regulate secretory pathways by yet unclear mechanisms [42, 43]. Identification of effectors involved in the regulation of the host secretory pathways will help us better understand both the bacterial pathogen and host cellular processes involved in infection, and thus further studies are needed.

Taken together, our study demonstrates that SdeA targets the Golgi and ubiquitinates Golgi tethering proteins GRASP55 and GRASP65, resulting in Golgi disruption and inhibition of secretory pathway. By revealing the biological consequences of PRubiquitination on Golgi proteins, our study provides a Golgi manipulation strategy, which Legionella utilizes to benefit bacterial infection. It will be interesting to study whether PR-ubiquitination confers additional versatile mechanisms to facilitate bacterial infection by verifying more substrates of SidE effectors in future.

\section{MATERIALS AND METHODS \\ Antibodies and reagents}

All reagents were from Sigma, Roche or Roth. The following antibodies were used: antibodies against HA (C29F4), GFP (sc-9996), GRASP65 (sc374423), from Santa Cruz; ubiquitin (P4BD) and ubiquitin (ab7254) from Cell signaling and Abcam respectively; mCherry (ab125096), Tubulin (ab6046), Calnexin (ab22595), Legionella (ab20943) from abcam; GM130 (D6B1), GAPDH (D16H11) from Cell signaling; GM130 (610823) from BD for 
IF only; GRASP55 (10598-1-AP) from proteintech, TGN46 (AHP500) from Biorad. Monoclonal Anti-HA-Agarose antibody (HA-7) was purchased from Sigma.

\section{Cloning and mutagenesis}

For protein expression in mammalian cells, GFP or mCherry tagged DupA wild-type EGFP-SdeA and catalytically defective mutants SdeA H277A and SdeA EEAA were generated as described previously [10]. SdeA plasmids were digested with BamHI and Xhol, then inserted into $\mathrm{mCherry-C1}$ vectors digested with BamHI and Xhol to generate $\mathrm{N}$ terminally mCherry tagged wild-type and mutated SdeA. Deletion of SdeA was designed according to the known structure and sequence prediction analyses. Truncated deletions Sde $A^{1-972}$ and Sde $A^{909-C}$ were amplified from full-length SdeA cDNA and digested with BamHI and Xhol. The digested DNA fragments were inserted into $\mathrm{pEGFP-C1}$ vectors digested with BamHI and Xhol. GFP or HA tagged GRASP55 and GRASP65-GFP were generated by PCR from GRASP55 or GRASP65 CDNA and digested with Xhol and BamHI or HindllI and Kpnl respectively, then inserted into pEGFP-N1 or pHA-N1 vector. For generation of the GRASP55 $7 \mathrm{~S}^{*}$ mutant, identified serines and adjacent serines S3, S4, S449, S451 were mutated to threonine to minimally effect the physio-chemical properties of these amino acids, in addition, S408, S409, S441 were mutated to alanine by site-directed mutagenesis. For protein expression in E. coli, SdeA was amplified from SdeA cDNA and digested with BamHI and Xhol. The digested DNA fragments were inserted into pGEX-6p-1 vector digested with BamHI and Xhol. GRASP55 and GRASP65 CDNA were amplified from mammalian vector and digested with Ndel and BamHI and cloned into pET15b and pGEX-6p-1 vector respectively. Serine to threonine or alanine mutations were generated by site-directed mutagenesis.

\section{Cell lines culture and transfection}

HEK293T, A549, COS7 cells were purchased from ATCC. Cells were cultured in high glucose Dulbecco's Modified Eagles Medium (DMEM) supplemented with $10 \%$ fetal bovine serum (FBS), $100 \mathrm{U} / \mathrm{mL}$ penicilin and $100 \mathrm{mg} / \mathrm{mL}$ streptomycin at $37^{\circ} \mathrm{C}, 5 \% \mathrm{CO}_{2}$ in a humidified incubator. Transfection was performed using polyethyleneimine (PEI) reagent or Genejuice (Merck).

\section{Legionella culture and infection}

Legionella strains were obtained from Dr. Zhao-Qing Luo lab (Purdue University). Cells were streaked and cultured at $37^{\circ} \mathrm{C}$ on N-(2-acetamido)-2aminoethanesulfonic acid (ACES)-buffered charcoal-yeast extract (BCYE) agar plates for 3 days, followed by inoculation and growth for $20 \mathrm{~h}$ in $3 \mathrm{~mL}$ CYE liquid media. Post-exponential Legionella with $\mathrm{OD}_{600}$ between 3.6-3.8 were used to infect A549 or HEK293T cells. HEK293T cells were transfected with FCYRII and GRASP55-GFP or GRASP65-GFP for $24 \mathrm{~h}$. Indicated Legionella strains were opsonized with antibody against Legionella $(1: 500)$ at $37^{\circ} \mathrm{C}$ for $30 \mathrm{~min}$ before infection. The HEK293T cells were infected with different Legionella strains at an $\mathrm{MOI}$ of 2 (for confocal imaging), or 10 (for Western blot) for the indicated time.

\section{SdeA mediated PR-ubiquitination reaction}

SdeA mediated PR-Serine ubiquitination in vitro reaction was done as previously described [13]. Briefly, $5 \mu \mathrm{M}$ GRASP proteins were incubated with $1 \mu \mathrm{M}$ of SdeA and 25 of $\mu \mathrm{M}$ ubiquitin in the presence of $200 \mu \mathrm{M}$ of $\mathrm{NAD}^{+}$in $40 \mu \mathrm{L}$ of reaction buffer $(50 \mathrm{mM} \mathrm{NaCl}$ and $50 \mathrm{mM}$ Tris, $\mathrm{pH} 7.5)$ for $1 \mathrm{~h}$ at $37^{\circ} \mathrm{C}$. Deubiquitination assay were performed by incubating PRubiquitinated proteins with $1 \mu \mathrm{g}$ of GST-DupA at $37^{\circ} \mathrm{C}$ for $1 \mathrm{~h}$ in reaction buffer $(150 \mathrm{mM} \mathrm{NaCl}, 50 \mathrm{mM}$ Tris- $\mathrm{HCl} \mathrm{pH} 7.5)$. The reaction products were analyzed by SDS-PAGE with Coomassie staining or western blotting using antibodies against GST (cell signaling technology), His (Roche), GRASP55 (Proteintech), GRASP65 (Sino biotech.), Ub (ab7254 from Abcam, 39365 from Cell signaling technology). To assess the PR-ubiquitination of GRASP55 and GRASP65 in cells, plasmids for expression of GRASPs-GFP, GFP-SdeA or mCherry-SdeA, were co-transfected into HEK293T cells, cells were then cultured at $37^{\circ} \mathrm{C}$ for $24 \mathrm{~h}$. Whole cell lysates were subjected to immunoprecipitation with GFP-trap beads and the products or the whole cell lysates were separated with SDS-PAGE and blotted with antibodies against GFP or GRASP proteins.

\section{Western blotting and immunoprecipitation}

Cell lysates or immunoprecipitated proteins were mixed with SDS sample buffer, heated at $95^{\circ} \mathrm{C}$ for $5 \mathrm{~min}$, centrifuged, and separated by Tris-Glycine
SDS-PAGE, and transferred to PVDF membrane (Millipore) at cold room. Blots were blocked with $5 \%$ nonfat milk for $1 \mathrm{~h}$ at room temperature and incubated with primary antibodies overnight at cold room or $2 \mathrm{~h}$ at room temperature and washed with TBST $(0.1 \%$ Tween 20 in TBS) three times. The blots were further incubated with secondary antibodies for $1 \mathrm{~h}$ at room temperature and washed 3 times with TBST. The blots were incubated with $\mathrm{ECL}$ reagents (advansta), and chemiluminescence was acquired with the Bio-Rad ChemiDoc system. For immunoprecipitation, HEK293T cells expressing GFP or HA-tagged proteins were lysed with mild immunoprecipitation buffer containing $150 \mathrm{mM} \mathrm{NaCl}, 50 \mathrm{mM}$ Tris- $\mathrm{HCl}, \mathrm{pH} 7.5,0.5 \%$ NP40, 1 mM PMSF, protease inhibitor cocktail (Sigma Aldrich), mixed with $10 \mu \mathrm{L}$ GFP-trap or HA antibody conjugated agarose, and incubated for $4 \mathrm{~h}$ in cold room with end to end rotation. Beads were washed 3 times in IP buffer containing $500 \mathrm{mM} \mathrm{NaCl}$. Proteins were eluted by resuspending with 2X SDS sample buffer followed by boiling for $5 \mathrm{~min}$ at $95^{\circ} \mathrm{C}$. Samples were then submitted to western blotting analysis.

\section{Protein expression and purification}

GRASP55 and GRASP65 cDNA were cloned into p15b and pGEX-6p-1 vector respectively. Full-length SdeA was cloned into pGEX-6P-1 vector. $E$. coli competent cells (NEB T7 express) were transformed with plasmid, colonies were inoculated and cultured in LB medium overnight at $37{ }^{\circ} \mathrm{C}$, The next day $5 \mathrm{~mL}$ culture was transferred to $1 \mathrm{~L}$ flask for further culture at $37^{\circ} \mathrm{C}$ until the $\mathrm{OD}_{600}$ reaches to $0.6-0.8$. Protein expression was induced by adding $0.5 \mathrm{mM} \mathrm{IPTG}$ and cells were further cultured overnight at $18^{\circ} \mathrm{C}$. The cells were harvested and the cell pellet was resuspended in lysis buffer $(300 \mathrm{mM} \mathrm{NaCl}, 50 \mathrm{mM}$ Tris- $\mathrm{HCl} \mathrm{pH} 7.5)$ followed with sonication and centrifuged at $13,000 \mathrm{rpm}$ to clarify the supernatant. Clarified lysates were then incubated with TALON beads or glutathione-S-Sepharose preequilibrated with washing buffer. Once eluted, proteins were further concentrated with filters and then purified by anion exchange chromatography on HitrapQ (GE Healthcare) and collected fractions were further loaded onto size exclusion column (Superdex 75 16/60, GE Healthcare). Proteins were concentrated and used for in vitro reaction.

\section{Identification of PR-ubiquitination serine sites on GRASP55} His-GRASP55 were purified from E. coli and PR-ubiquitinated by SdeA in vitro. Samples were prepared as previously described [10, 13]. Briefly, added urea buffer containing $8 \mathrm{M}$ urea, $0.1 \mathrm{M}$ Tris, $\mathrm{pH} 7.5$ to the reaction mixture to a final volume of $200 \mu \mathrm{L}$, the reactions were then transferred to $30 \mathrm{kDa}$ filter (Amicon Ultra, $0.5 \mathrm{~mL}$, Merck) and washed 3 times with $200 \mu \mathrm{L}$ of urea buffer by centrifugation to remove free ubiquitin. Proteins were washed 2 times with $50 \mathrm{mM} \mathrm{ABC}, \mathrm{pH} 7.5$, then digested with trypsin in 50 $\mathrm{mM} \mathrm{ABC} \mathrm{pH} 7.5$ at trypsin to protein ratio 1:50 for $6 \mathrm{~h}$ and subsequently desalted by $\mathrm{C} 18$ and analyzed by LC MS/MS.

\section{Data quantification}

Data shown in Figs. 2B, 2D, 6D, 8B, 8D were analyzed with GraphPad Prism 5.0. Three independent experiments were performed, $p$ values were determined using unpaired two-tailed $t$ test, ${ }^{* * *}, * * * *$ and ns represent $p<$ $0.0001, p<0.01, p<0.05$ and not significant respectively. For Fig. $2 \mathrm{~B}$ and $2 \mathrm{D}$, more than $70 \mathrm{SdeA}$ transfected or Legionella infected cells were examined from 3 replicates of each condition. Values of percentage of cells with fragmented Golgi were input into GraphPad Prism, and analyzed. For Fig. 6D, Golgi areas of more than 60 cells from 3 replicates of each condition were measured with ImageJ software. For Fig. 8B, data represents 30 cells taken from 3 independent experiments. For Fig. 8D, gray values of VSVG bands shown as Fig. $8 \mathrm{C}$ from 3 replicates were measured with ImageJ.

\section{VSVG trafficking assay}

HEK293T or A549 cells were co-transfected with VSVG-GFP and FcyRII or transfected with VSVG-GFP respectively and cultured at $37^{\circ} \mathrm{C}$ for $24 \mathrm{~h}$ to express the proteins before being transferred to $40^{\circ} \mathrm{C}$. After $16 \mathrm{~h}$ incubation at $40^{\circ} \mathrm{C}$, cycloheximide was added into medium to inhibit further protein synthesis, after $2 \mathrm{~h}$ treatment cells were infected with Legionella for another $2 \mathrm{~h}$ then washed 3 times with PBS and cultured with fresh medium at $32^{\circ} \mathrm{C}$ to remove the bacteria outside host cells, and then moved to $32^{\circ} \mathrm{C}$ for different time points to release VSVG from ER. A549 cells were fixed and VSVG trafficking was acquired with confocal microscopy after immunofluorescence staining. DAPI marks nucleus and cytosolic bacteria. For calculating Manders coefficient in FIJ, ROIs of 
$30 \mu \mathrm{m}^{2}$ are chosen from the perinuclear region containing the Golgi marked by GM130. Manders coefficient is calculated using Coloc2 plugin in FIJI and denotes fraction of VSVG-GFP pixels that is positive for GM130. For EndoH cleavage assay, HEK293T cells were lysed with lysis buffer containing $1 \%$ SDS, $50 \mathrm{mM}$ Tris, $\mathrm{pH}$ 8.0. Benzonase was added to reduce the viscosity caused by released DNA. Cell lysates were mixed with denaturing buffer then boiled for $10 \mathrm{~min}$ at $95^{\circ} \mathrm{C}$. Denatured proteins were incubated with EndoH for $3 \mathrm{~h}$ at $37^{\circ} \mathrm{C}$ to cleave the EndoH sensitive form of glycosylation, final products were separated with SDS-PAGE and the EndoH-caused band shift was analyzed by blotting GFP.

\section{Immunofluorescence}

HEK293T, COS7, or A549 cells were seeded on a coverslip in 12-well plates and cultured in $\mathrm{CO}_{2}$ incubator. Next day cells were transfected with plasmids encoding SdeA. The immunostaining was performed following the protocol previously described [10]. Briefly, cells were washed once with PBS, pH 7.4, and fixed with 4\% paraformaldehyde (PFA) in PBS for 10 min at room temperature. Cells were washed again with PBS 2 times, then permeabilized with $0.1 \%$ saponin in PBS for $10 \mathrm{~min}$, and blocked with blocking buffer containing $0.1 \%$ saponin and $2 \%$ BSA in PBS for $1 \mathrm{~h}$ at room temperature. Cells were stained with antibodies diluted in blocking buffer overnight at $4{ }^{\circ} \mathrm{C}$ and washed with PBS three times next day. Cells were further incubated with Alexa Flour dyes-conjugated secondary antibodies for $1 \mathrm{~h}$ at room temperature in the dark and washed with PBS and incubated with DAPI in PBS, followed with further 2 times washing with PBS. Confocal imaging was performed using the Zeiss LSM780 microscope system. Images were analyzed with Fiji software.

\section{DNA-PAINT super-resolution Imaging}

COS7 cells were fixed with prewarmed $\left(37^{\circ} \mathrm{C}\right) \quad 4 \%$ methanol-free formaldehyde (Sigma-Aldrich, Germany) in PBS for $10 \mathrm{~min}$ followed by three washing steps with PBS. Cells were quenched with $0.1 \% \mathrm{NaBH}_{4}$ (Sigma Aldrich, Germany) for $7 \mathrm{~min}$ in PBS and washed thrice with PBS. Fixed cells were permeabilized and blocked in permeabilization/blockingbuffer, followed by incubation of primary antibodies against GM130 and Golgin 97 in permeabilization/blocking buffer for $90 \mathrm{~min}$. Washed cells were then incubated with DNA-labeled secondary antibodies for $1 \mathrm{~h}$ and washed again. Finally, samples were post-fixed using $4 \%$ methanol-free formaldehyde for $10 \mathrm{~min}$ at room-temperature followed by three washing steps with PBS. For sequential DNA-PAINT imaging, $125 \mathrm{~nm}$ gold-beads (Nanopartz, USA) were used as fiducial markers. Exchange DNA-PAINT measurements were performed at the N-STORM super-resolution microscopy system (Nikon, Japan) equipped with an oil immersion objective (Apo, 100x, NA 1.49) and an EMCCD camera (DU-897U-CS0-\#BV, Andor Technology, Ireland).

\section{REFERENCES}

1. Hershko A, Ciechanover A, Varshavsky A. The ubiquitin system. Nat Med. 2000;6:1073-81.

2. Ben-Neriah Y. Regulatory functions of ubiquitination in the immune system. Nat Immunol. 2002;3:20-6.

3. Donaldson KM, Yin $H$, Gekakis N, Supek F, Joazeiro CAP. Ubiquitin signals protein trafficking via interaction with a novel ubiquitin binding domain in the membrane fusion regulator, Vps9p. Curr Biol. 2003;13:258-62.

4. Dikic I. Proteasomal and autophagic degradation systems. Annu Rev Biochem. 2017;86:193-224.

5. Rape M. Post-translational modifications: ubiquitylation at the crossroads of development and disease. Nat Rev Mol Cell Biol. 2018;19:59-70.

6. Bomberger JM, Ye S, MacEachran DP, Koeppen K, Barnaby RL, O'Toole GA, et al. A Pseudomonas aeruginosa toxin that hijacks the host ubiquitin proteolytic system. PLoS Pathog. 2011;7:e1001325.

7. Hicks SW, Galán JE. Exploitation of eukaryotic subcellular targeting mechanisms by bacterial effectors. Nat Rev Microbiol. 2013;11:316-26.

8. Maculins T, Fiskin E, Bhogaraju S, Dikic I. Bacteria-host relationship: ubiquitin ligases as weapons of invasion. Cell Res 2016;26:499-510.

9. Qiu J, Sheedlo MJ, Yu K, Tan Y, Nakayasu ES, Das C, et al. Ubiquitination independent of E1 and E2 enzymes by bacterial effectors. Nature. 2016;533:120-4.

10. Bhogaraju S, Kalayil S, Liu Y, Bonn F, Colby T, Matic I, et al. Phosphoribosylation of ubiquitin promotes serine ubiquitination and impairs conventional ubiquitination. Cell. 2016;167:1636-1649.e13.

11. Akturk A, Wasilko DJ, Wu X, Liu Y, Zhang Y, Qiu J, et al. Mechanism of phosphoribosyl-ubiquitination mediated by a single legionella effector. Nature. 2018;557:729-45.
12. Dong $Y$, Mu Y, Xie $Y$, Zhang $Y$, Han $Y$, Zhou $Y$, et al. Structural basis of ubiquitin modification by the Legionella effector SdeA. Nature. 2018;557:674-8.

13. Kalayil S, Bhogaraju S, Bonn F, Shin D, Liu Y, Gan N, et al. Insights into catalysis and function of phosphoribosyl-linked serine ubiquitination. Nature. 2018;557:734-8.

14. Wang $Y$, Shi M, Feng $H$, Zhu Y, Liu S, Gao A, et al. Structural insights into noncanonical ubiquitination catalyzed by SidE. Cell. 2018;173:1231-43.

15. Shin D, Mukherjee R, Liu Y, Gonzalez A, Bonn F, Liu Y, et al. Regulation of phosphoribosyl-linked serine ubiquitination by deubiquitinases DupA and DupB. Mol Cell. 2020;77:164-79.

16. Knodler LA, Ibarra JA, Pérez-Rueda E, Yip CK, Steele-Mortimer O. Coiled-coil domains enhance the membrane association of Salmonella type III effectors. Cell Microbiol. 2011;13:1497-517.

17. Kotewicz KM, Ramabhadran V, Sjoblom N, Vogel JP, Haenssler E, Zhang M, et al. A single legionella effector catalyzes a multistep ubiquitination pathway to rearrange tubular endoplasmic reticulum for replication. Cell Host Microbe. 2017;21:169-81.

18. Qiu J, Yu K, Fei X, Liu Y, Nakayasu ES, Piehowski PD, et al. A unique deubiquitinase that deconjugates phosphoribosyl-linked protein ubiquitination. Cell Res. 2017;27:865-81.

19. Jeong KC, Sexton JA, Vogel JP. Spatiotemporal regulation of a Legionella pneumophila T4SS substrate by the metaeffector SidJ. PLoS Pathog. 2015;11:1-22.

20. Grond R, Veenendaal T, Duran JM, Raote I, van Es JH, Corstjens S, et al. The function of GORASPs in Golgi apparatus organization in vivo. J. Cell Biol. 2020;219:e202004191.

21. Sohda M, Misumi Y, Yamamoto A, Yano A, Nakamura N, Ikehara Y. Identification and characterization of a novel golgi protein, GCP60, that interacts with the integral membrane protein giantin. J Biol Chem. 2001;276:45298-306.

22. Jarvela T, Linstedt AD. Golgi GRASPs: moonlighting membrane tethers. Cell Health Cytoskelet. 2012;4:37-47.

23. Rabouille C, Linstedt AD. GRASP: a multitasking tether. Front Cell Dev Biol. 2016:4:1-8.

24. Feinstein TN, Linstedt AD. GRASP55 regulates Golgi ribbon formation. Mol Biol Cell. 2008;19:2696-707.

25. Bekier ME, Wang L, Li J, Huang H, Tang D, Zhang X, et al. Knockout of the Golgi stacking proteins GRASP55 and GRASP65 impairs Golgi structure and function. Mol Biol Cell. 2017;28:2833-42.

26. Heuer D, Lipinski AR, Machuy N, Karlas A, Wehrens A, Siedler F, et al. Chlamydia causes fragmentation of the Golgi compartment to ensure reproduction. Nature. 2009:457:731-5.

27. $\mathrm{Xu} \mathrm{L}$, Luo ZQ. Cell biology of infection by Legionella pneumophila. Microbes Infect. 2013;15:157-67.

28. Scidmore MA, Fischer ER, Hackstadt T. Sphingolipids and glycoproteins are differentially trafficked to the Chlamydia trachomatis inclusion. J Cell Biol. 1996;134:363-74.

29. De Jong AS, Visch HJ, De Mattia F, Van Dommelen MM, Swarts HG, Luyten T, et al. The coxsackievirus 2B protein increases efflux of ions from the endoplasmic reticulum and Golgi, thereby inhibiting protein trafficking through the Golgi. J Biol Chem. 2006;281:14144-50.

30. Burke B, Matlin K, Bause E, Legler G, Peyrieras N, Ploegh H. Inhibition of N-linked oligosaccharide trimming does not interfere with surface expression of certain integral membrane proteins. EMBO J. 1984;3:551-6.

31. Ernst AM, Syed SA, Zaki O, Bottanelli F, Zheng H, Hacke M, et al. S-palmitoylation sorts membrane cargo for anterograde transport in the Golgi. Dev Cell. 2018;47:479-93.

32. Wang $Y$, Satoh A, Warren G. Mapping the functional domains of the Golgi stacking factor GRASP65. J Biol Chem. 2005;280:4921-8.

33. Bian $Y$, Song $C$, Cheng $K$, Dong $M$, Wang F, Huang J, et al. An enzyme assisted RPRPLC approach for in-depth analysis of human liver phosphoproteome. J Proteom. 2014;96:253-62.

34. Kim J, Noh SH, Piao H, Kim DH, Kim K, Cha JS, et al. Monomerization and ER relocalization of GRASP is a requisite for unconventional secretion of CFTR. Traffic. 2016;17:733-53.

35. Truschel ST, Zhang M, Bachert C, Macbeth MR, Linstedt AD. Allosteric regulation of GRASP protein-dependent golgi membrane tethering by mitotic phosphorylation. J Biol Chem. 2012;287:19870-5.

36. Weber S, Steiner B, Welin A, Hilbi H. Legionella-containing vacuoles capture Ptdlns(4)P-rich vesicles derived from the golgi apparatus. MBio. 2018;9: e02420-18.

37. Urwyler S, Nyfeler Y, Ragaz C, Lee H, Mueller LN, Aebersold R, et al. Proteome analysis of Legionella vacuoles purified by magnetic immunoseparation reveals secretory and endosomal GTPases. Traffic. 2009;10:76-87.

38. Schmölders J, Manske C, Otto A, Hoffmann C, Steiner B, Welin A, et al. Comparative proteomics of purified pathogen vacuoles correlates intracellular replication of Legionella pneumophila with the small GTPase ras-related protein 1 (Rap1). Mol Cell Proteom. 2017;16:622-41. 
39. Wan M, Sulpizio AG, Akturk A, Beck WHJ, Lanz M, Faça VM, et al. Deubiquitination of phosphoribosyl-ubiquitin conjugates by phosphodiesterase-domain-containing Legionella effectors. Proc Natl Acad Sci USA. 2019;116:23518-26.

40. Nagashima S, Tábara LC, Tilokani L, Paupe V, Anand H, Pogson JH, et al. Golgiderived $\mathrm{Pl}(4) \mathrm{P}$-containing vesicles drive late steps of mitochondrial division. Sci (80-). 2020;367:1366-71.

41. Escoll P, Song OR, Viana F, Steiner B, Lagache T, Olivo-Marin JC, et al. Legionella pneumophila modulates mitochondrial dynamics to trigger metabolic repurposing of infected macrophages. Cell Host Microbe. 2017;22:302-16.

42. Nagai H, Kagan JC, Zhu X, Kahn RA, Roy CR. A bacterial guanine nucleotide exchange factor activates ARF on Legionella phagosomes. Sci (80-). 2002;295:679-82.

43. Machner MP, Isberg RR. Targeting of host Rab GTPase function by the intravacuolar pathogen Legionella pneumophila. Dev Cell. 2006;11:47-56.

\section{ACKNOWLEDGEMENTS}

We thank Zhao-Qing Luo for the kind gift of Legionella strains wild-type and $\Delta$ sidEs, Hubert Hilbi for the kind gift of Legionella expressing dsRed, Yanzhuang Wang for the kind gift of GRASP55/65 Knockout HeLa cell line; Mohit Misra, Anne-Claire Jacomin and Alexandra Stolz for discussion and critical reading of the manuscript.

\section{FUNDING STATEMENT}

This project was funded by European Research Council (ERC) under the European Union's Horizon 2020 research and innovation programme (ID, grant agreement No 742720), and LOEWE Main Research Focus DynaMem of the German Federal State of Hesse (III L6-519/03/03.001-(O006), to ID). Work in Ivan Matic's laboratory was funded by the Deutsche Forschungsgemeinschaft (DFG, German Research Foundation) under Germany's Excellence Strategy-CECAD, EXC 2030-390661388 (to IM), and the EMBO Young Investigator Programme (to IM). MH and MG acknowledge funding by the Deutsche Forschungsgemeinschaft (DFG, German Research Foundation)-Project-ID 253130777-SFB 1177. Open Access funding enabled and organized by Projekt DEAL

\section{AUTHOR CONTRIBUTIONS}

$\mathrm{YL}$ and ID designed the study and experiments. RM performed the VSVG trafficking experiment. $\mathrm{FB}, \mathrm{TC}$, and $\mathrm{IM}$ performed mass spectrometry experiments and dataanalysis. YL performed biochemical, cell biological and bacterial infection experiments and data-analysis. MG and MH performed DNA-PAINT super-resolution microscopy imaging. $\mathrm{YL}$ and ID wrote the manuscript and all authors commented on it.

\section{COMPETING INTERESTS}

The authors declare no competing interests.

\section{ETHICS STATEMENT}

This study did not require ethical approval

\section{ADDITIONAL INFORMATION}

Supplementary information The online version contains supplementary material available at https://doi.org/10.1038/s41418-021-00830-y.

Correspondence and requests for materials should be addressed to I.D.

Reprints and permission information is available at http://www.nature.com/ reprints

Publisher's note Springer Nature remains neutral with regard to jurisdictional claims in published maps and institutional affiliations.

(i) Open Access This article is licensed under a Creative Commons Attribution 4.0 International License, which permits use, sharing, adaptation, distribution and reproduction in any medium or format, as long as you give appropriate credit to the original author(s) and the source, provide a link to the Creative Commons license, and indicate if changes were made. The images or other third party material in this article are included in the article's Creative Commons license, unless indicated otherwise in a credit line to the material. If material is not included in the article's Creative Commons license and your intended use is not permitted by statutory regulation or exceeds the permitted use, you will need to obtain permission directly from the copyright holder. To view a copy of this license, visit http://creativecommons. org/licenses/by/4.0/.

C The Author(s) 2021 